\title{
An Immune-Related IncRNA Signature to Predict the Biochemical Recurrence and Immune Landscape in Prostate Cancer
}

\author{
Guian Zhang' \\ Yong Luo iD ${ }^{2}$ \\ 'School of Medicine, South China \\ University of Technology, Guangzhou, \\ 510006, People's Republic of China; \\ ${ }^{2}$ Department of Urology, the Second \\ People's Hospital of Foshan, Affiliated \\ Foshan Hospital of Southern Medical \\ University, Foshan, 528000, People's \\ Republic of China
}

Purpose: This study aims to construct an immune-related signature to provide comprehensive insights into the immune landscape of prostate cancer, which can predict biochemical recurrence $(\mathrm{BCR})$ and clinical treatment.

Methods: Based on The Cancer Genome Atlas (TCGA) dataset, a signature constructed by DEirlncRNAs pairs was determined. The receiver operating characteristic curve analysis, Kaplan-Meier analysis, nomogram, and decision curve analysis were used to analyze it. Then, immunophenoscore (IPS), immune cell infiltration, tumor mutation burden (TMB), and immune function were investigated. Finally, we evaluated the role of the signature in medical treatment.

Results: A signature constructed by 10 valid DEirlncRNAs pairs was identified in the training set and validated well in the testing and entire set. The signature was a reliable and independent prognostic indicator to predict the BCR of prostate cancer, which was better than the clinicopathological characteristics. After dividing the patients into low- and highrisk groups by median value, we found that the high-risk group had shorter BCR-free time and higher TMB levels. Furthermore, the high-risk group was negatively associated with plasma $\mathrm{B}$ cells and $\mathrm{CD}+8 \mathrm{~T}$ cells. IPS and immune functions, such as immune checkpoints and human leukocyte antigen, were significantly different between the two groups. Low-risk group was more sensitive to endocrine therapy and immunotherapy, while high-risk group was more inclined to targeted drugs. Both groups had their own sensitive chemotherapy.

Conclusion: We established a novel signature to predict BCR and validated its role in the immune landscape of prostate cancer, which could help patients receive personalized medical treatment.

Keywords: prostate cancer, lncRNA, biochemical recurrence, TMB, immune landscape, medical treatment

\section{Introduction}

Prostate cancer, a high morbidity solid tumor, is the second most frequent cancer and the fifth leading cause of cancer death among men in $2020 .{ }^{1,2}$ Currently, the clinical treatment for patients with localized prostate cancer usually adopts radical prostatectomy, which provides a certain degree of relief. ${ }^{3}$ However, it is not sufficient, as about $20-40 \%$ of patients will experience biochemical prostatespecific antigen recurrence after radical prostatectomy. ${ }^{4}$ Until now, a lot of studies have indicated that the progression of prostate cancer was affected by many factors, such as PSA level, pathological stage, and Gleason score., ${ }^{5,6}$ Due to the
Department of Urology, the Second People's Hospital of Foshan, Affiliated Foshan Hospital of Southern Medical University, 78 Weiguo Road, Foshan, 528000 , People's Republic of China

Tel +86-I5625093895

Fax +86-0757-88032009

Email luomou5@outlook.com 
heterogeneous nature and biochemical recurrence (BCR) of prostate cancer, the existing strategies for prognosis judgment are nuanced and varied, which fail to meet the needs of every patient. Prostate cancer patients with BCR are the main challenge faced by clinicians. Failure to correctly identify these cases will lead to disease progression without the most appropriate management. New recommendations on the prognostic models and novel features for predicting the prognosis of prostate cancer are urgently required for modern clinicians to manage BCR.

The tumor microenvironment consists of cancer cells, stromal cells, extracellular matrix, and immune cells, such as T lymphocytes, macrophages, and neutrophils. ${ }^{7}$ It is well known that the immune system processes and abnormal immune response in the tumor microenvironment play a crucial role in the recurrence, progression, and metastasis of tumors. ${ }^{8}$ Multiple characteristics had been shown to affect the tumor microenvironment, including tumor mutation burden (TMB) and human leukocyte antigen (HLA). ${ }^{9-11}$ In addition, chemotherapy, radiotherapy, and targeted drugs could alter the tumor environment, which made the tumor environment elusive in the treatment process. ${ }^{12,13}$ Hence, it was essential to consider the immune-related factors associated with the BCR of prostate cancer patients and medical treatment.

Long noncoding RNAs (lncRNAs), accounting for approximately $80 \%$ of the human transcriptome, refer to a series of RNA molecules with transcripts longer than 200 nucleotides. ${ }^{14,15}$ Although lncRNAs cannot code for proteins, they can exert biological functions by interacting with DNA, RNA, and proteins to regulate gene expression. ${ }^{16-18}$ Increasing research reported that IncRNAs, as new transcriptional regulators, were not only related to normal life development but also contributed to the occurrence, progression, and prognosis of human diseases. ${ }^{19-22}$ LncRNAs were expected to be the signatures of tumor immune infiltrates, which deserved useful predictive and prognostic value in the diagnosis, evaluation, and treatment of cancer. The latest research showed that IncRNA could activate the immune system and immune response by altering the expression of immune-related genes, and then affect the malignant phenotype of cancer by changing the tumor immune microenvironment. ${ }^{15,23-27}$ Sun et $\mathrm{al}^{28}$ identified five immune-related lncRNAs (irlncRNAs) and constructed a five-lncRNAs signature to predict the prognosis for renal clear cell carcinoma. Zhu et al ${ }^{29}$ integrated eight irlncRNAs and clinical nomograms to evaluate the survival of patients with esophageal squamous cell carcinoma. Therefore, it was significant to explore irlncRNAs to predict the prognosis of prostate cancer patients and further guide appropriate individual treatment strategies.

In this study, we had identified a signature constructed by 10 DEirlncRNAs pairs in the training set and validated it well in the testing set and the entire The Cancer Genome Atlas (TCGA) set. The signature had strong predictive power in the BCR of prostate cancer and served as an independent prognostic factor. Then, we found that the signature was closely related to the clinicopathological characteristics and was superior to the traditional clinical features. In addition, immunophenoscore (IPS), TMB, immune cell infiltration, and immune function were highly correlated with the signature. Finally, this signature was also of great value in evaluating the therapeutic effects of chemotherapy, endocrine therapy, and targeted drug. In summary, we established a novel signature in predicting the BCR of prostate cancer, which might be of great help to guide clinical treatment in the future.

\section{Materials and Methods Dataset Preparation}

The fragments per kilobase of per million (FPKM) of prostate cancer transcriptome and corresponding clinical information were retrieved from the TCGA program (https://tcga-data.nci.nih.gov/tcga/). To distinguish between mRNAs and IncRNAs, the GTF files were downloaded from the Ensembl (http://asia.ensembl.org). Immune-related genes (ir-genes) were extracted from the ImmPort database (http://www.immport.org). Meanwhile, mutation data of prostate cancer patients were obtained from the TCGA database as mutation annotation format (MAF) files. The inclusion criteria were patients with both BCR events and recorded BCR time. Sample with no BCR events, no recorded $\mathrm{BCR}$ time, or no clinical information were considered as exclusion criteria.

\section{Identification of Immune-Related IncRNA in Prostate Cancer}

IrlncRNAs were identified by correlation analysis between ir-genes and all lncRNAs (Pearson correlation coefficient $>0.5, \mathrm{p}<0.001)$. The differentially expressed irlncRNAs were identified by limma $\mathrm{R}$ package setting criterion as | $\log \mathrm{FC} \mid \geq 2$ along with the false discovery rate (FDR) $<0.05{ }^{30}$ 


\section{Construction and Validation of the Signature}

We used the $\mathrm{R}$ software package "caret" to randomly divide the entire TCGA set into a training set and a testing set. The training set was assigned to build the signature, and the entire set and testing set were used to verify the established model. To construct a 0 - or -1 matrix by cycling single pairs of DEirlncRNAs, if the expression level of the previous lncRNA was higher than the expression level of the latter lncRNA, the output was 1 ; otherwise, the output was 0 . When the expression of IncRNA pairs was 0 or 1 , no relationship between the pairing and $\mathrm{BCR}$ was considered. An effective match was that the number of lncRNAs pairs expressing as 0 or 1 accounted for more than $20 \%$ of the total pairs. The least absolute shrinkage and selection operator (Lasso) was performed to further sort with 10-fold cross-validation. Finally, the multiple stepwise Cox regression method was used to select the DEirlncRNAs pairs for constructing the risk model.

\section{Prediction Analysis of the Signature}

The area under curve (AUC) value of each model was calculated and plotted as a curve. ROC curves were used to assess the sensitivity and specificity of the model-based approach for predicting BCR. The formula used to calculate the risk score was as follows: Risk Score $=\sum^{n k} \beta \mathrm{iSi}$. The low- risk and high-risk groups were divided ${ }^{i}$ by the median value of risk score. The survival curves were used to assess the BCR of low- and high-risk groups by the method of Kaplan-Meier. The specific risk score values of each sample in the model were visualized using $\mathrm{R}$ tools. The $\mathrm{R}$ packages adopted in these steps included timeROC, survival, and survminer. ${ }^{31}$ Univariate and multivariate Cox regression analysis were used to confirm the potential of the signature as an independent prognostic predictor. A forest plot was used to depict the results of the $\mathrm{R}$ survival packages.

\section{Construction and Evaluation of a Predictive Nomogram}

Nomogram was set up by combining the risk score and clinical characteristics including age, grade, and stage to predict the BCR-free time in 1, 2, and 3 years. Calibration curves were utilized to prove the consistency between the actual results and the model predicted results. In order to compare the established nomogram with the traditional clinical factors, we also performed a decision curve analysis (DCA) to evaluate the clinical practicality of the nomogram.

\section{Exploration of Immune Status and IPS Analysis}

We used R package "maftools" to analyze and evaluate the information of TMB. ${ }^{32}$ Spearman correlation analysis was used to analyze the relationship between the risk score and immune infiltrating cells, which was shown in the lollipop graph. A relative immune cell abundance was assessed from the gene expression profile using the CIBERSORT, TIMER, CIBERSORT-ABS, QUANTISEQ, MCPCOUNTER, XCELL, and EPIC algorithms. The Wilcoxon sign-rank test was used to analyze the difference in the content of immune infiltrating cells between the low-risk group and the high-risk group, which was shown in the box diagram. In addition, single-sample gene set enrichment (ssGSEA) was applied to quantify the infiltrating immune cells and the immune function between the two groups. ${ }^{33}$ The process was performed using the R GSVA, ${ }^{34}$ limma, GSEABase, ggpubr, and reshape2 packages.

The stromal scores, immune scores, ESTIMATE scores, and tumor purity were calculated using the ESTIMATE algorithm. The IPS of prostate cancer patients was obtained from the TCIA (https://tcia.at/). Determined by the four factors of effector cells, immunosuppressive cells, immunomodulators, and MHC molecules, IPS was positively correlated with immunogenicity.

\section{Identify the Sensitivity of Medical Treatment}

We evaluated the IC50 for common agents in the TCGA program. The difference in IC50 between the low-risk and high-risk groups was compared by Wilcoxon signed-rank test, and the results were shown as box plots obtained using pRRophetic and ggplot2 of R packages. ${ }^{35}$

\section{Statistical Analysis}

All statistical analyses were applied using $\mathrm{R}$ version 3.6.3 (https://www.r-project.org), ${ }^{36}$ and some related packages were applied to all statistical analyses. The mean \pm standard deviation was used to describe continuous variables in a normal distribution. $\mathrm{P}<0.05$ was considered the significantly statistical difference. 


\section{Results}

Analysis of Immune-Related IncRNAs (irlncRNAs) and Differentially Expressed irlncRNAs (DEirlncRNAs)

The detailed workflow of the present study is summarized in Figure 1. First, the clinical information of a total of 499 prostate cancer samples and 52 paired adjacent normal samples as well as their transcriptome profiling data were obtained from TCGA database. Then, retrieving gene transfer format (GTF) file from Ensembl, we annotated the data and performed co-expression analysis between known immune-related genes (ir-genes) and lncRNAs. At last, 795 irlncRNAs were screened out by co-expression analysis (Table S1), and then a total of 29 irlncRNAs were identified as DEirlncRNAs (Figure 2A), of which 25 were up-regulated and 4 were down-regulated (Figure 2B).

\section{Constructing DEirlncRNAs Pairs as a Prognostic Signature for Prostate Cancer}

Among 29 DEirlncRNAs, 269 pairs of effective DEirlncRNAs were identified by the iterative loop method

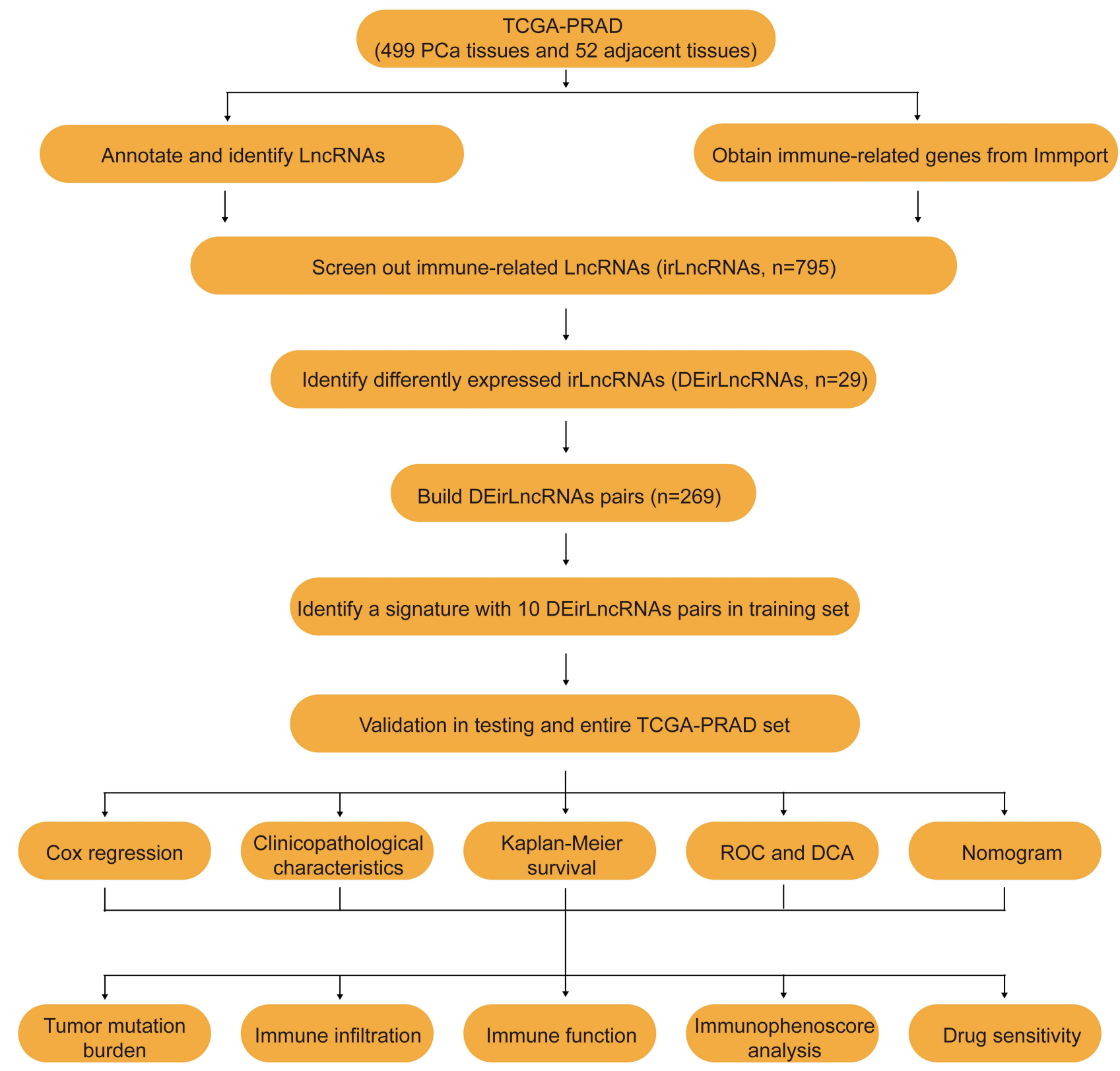

Figure I The flow diagram of this study. 

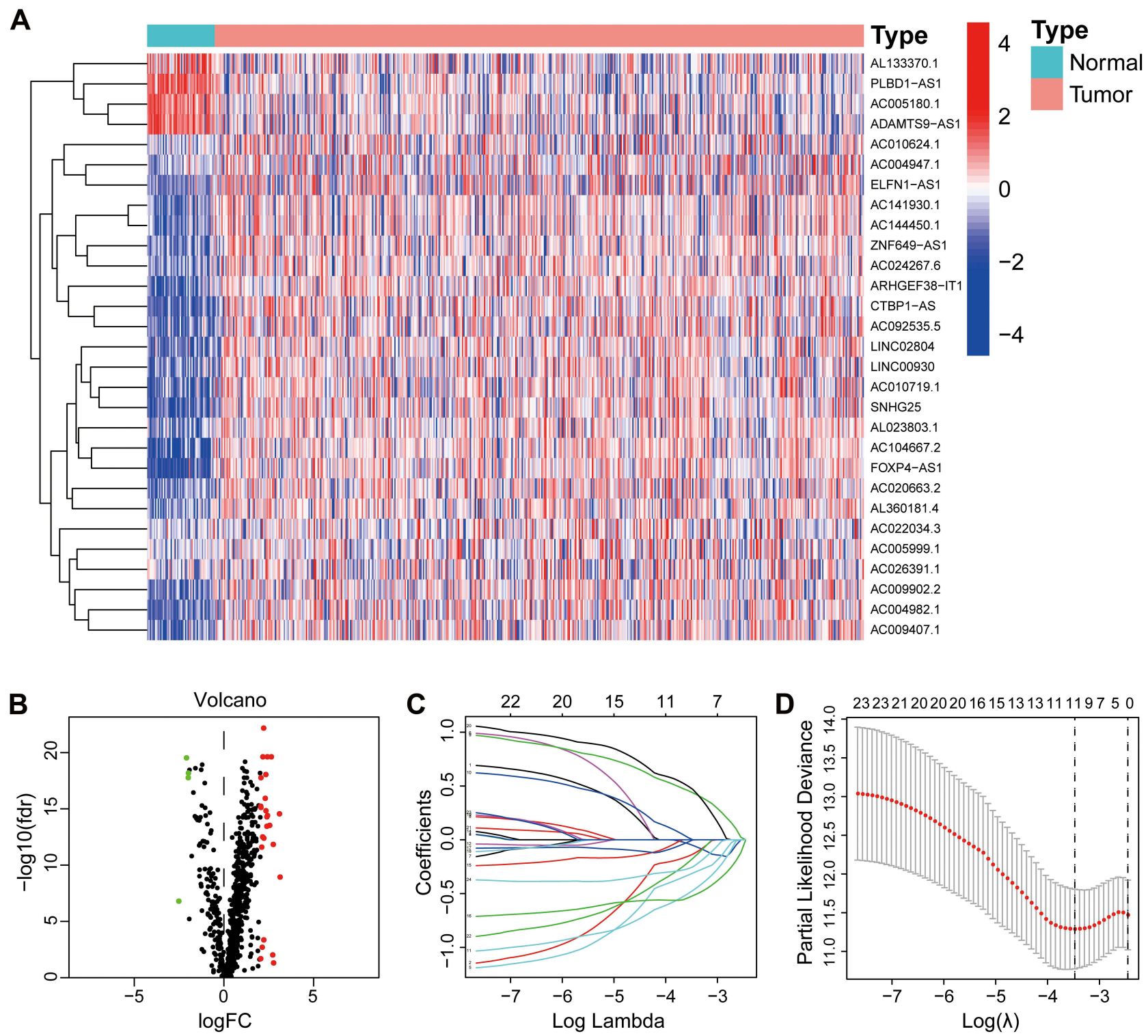

Figure 2 Construction of a prognostic signature for prostate cancer. The heatmap (A) and volcano (B) showed the DEirlncRNAs in TCGA database. (C) Process of variable selection in LASSO regression with 10 -fold cross-validation. (D) Confidence interval in every lambda of LASSO regression.

and 0 or 1 matrix screening. The entire TCGA set was randomized into the training set $(\mathrm{n}=214)$ and the testing set $(\mathrm{n}=$ 213). The clinical characteristics of both two sets are displayed in Table 1, and no significant differences in clinical properties were detected between them. To avoid overfitting the prognostic signature, the target DEirlncRNAs pairs were selected based on the Lasso regression model in the training set (Figure $2 \mathrm{C}$ and $\mathrm{D}$ ). The best values of the penalty parameter were determined by 10 -fold cross-validation. Then, adopting multiple regression analysis by stepwise method, 10 DEirlncRNAs pairs were selected and included in Cox proportional hazards models based on the BCR data of samples in the TCGA training set. (Figure S1A and $\underline{B}$ ).

\section{Evaluation of DEirlncRNAs Pairs as a Robust Predictive Signature in the Training Set}

Patients in the training set were divided into the low- and high-risk groups based on the median value of the risk score as the cut-off value. The risk curve and scatterplot were conducted to illustrate the relationship between the signature and $\mathrm{BCR}$ in the training set (Figure $3 \mathrm{~A}$ and $\mathrm{B}$ ). 
Table I Clinical Characteristics of Prostate Cancer Patients in Each Set

\begin{tabular}{|c|c|c|c|c|}
\hline Characteristics & Entire Set $(n=427)$ & Training Set $(n=214)$ & Testing Set $(n=213)$ & $P$-value \\
\hline \multicolumn{5}{|l|}{ Age (years) } \\
\hline$\leq 65$ & 306 (7I.7\%) & I 48 (69.2\%) & I 58 (74.2\%) & 0.250 \\
\hline$>65$ & $12 \mid(28.3 \%)$ & $66(30.8 \%)$ & 55 (25.8\%) & \\
\hline \multicolumn{5}{|l|}{ Tumor Grade } \\
\hline GI-G3 & $243(56.9 \%)$ & $120(56.1 \%)$ & 123 (57.7\%) & 0.727 \\
\hline G4-G5 & I84 (43.1\%) & 94 (43.9\%) & $90(42.3 \%)$ & \\
\hline \multicolumn{5}{|l|}{ Tumor Stage } \\
\hline Stage I-II & 209 (48.9\%) & $102(47.7 \%)$ & 107 (50.2\%) & 0.962 \\
\hline Stage III-IV & I0I (23.7\%) & 49 (22.9\%) & $52(24.4 \%)$ & \\
\hline Unknown & II (27.4\%) & $63(29.4 \%)$ & $54(25.4 \%)$ & \\
\hline \multicolumn{5}{|l|}{ BCR status } \\
\hline BCR-free & $369(86.4 \%)$ & $184(86.0 \%)$ & $185(86.9 \%)$ & 0.792 \\
\hline$B C R$ & $58(\mid 3.6 \%)$ & $30(14.0 \%)$ & $28(13.1 \%)$ & \\
\hline \multicolumn{5}{|c|}{ Tumor invasion ( $\mathrm{T}$ ) } \\
\hline $\mathrm{TI}-2$ & 307 (71.9\%) & I53 (7|.5\%) & 154 (72.3\%) & 0.911 \\
\hline T3-4 & 49 (II.5\%) & 24 (II.2\%) & 25 (II.7\%) & \\
\hline Unknown & $7 \mid(16.6 \%)$ & $37(17.3 \%)$ & $34(16.0 \%)$ & \\
\hline \multicolumn{5}{|l|}{ Lymph node (N) } \\
\hline No & 304 (7I.2\%) & 148 (69.1\%) & I56 (73.2\%) & 0.678 \\
\hline $\mathrm{NI}$ & $68(I 5.9 \%)$ & $35(16.4 \%)$ & 33 (I5.5\%) & \\
\hline Unknown & 55 (I2.9\%) & 31 (14.5\%) & 24 (II.3\%) & \\
\hline \multicolumn{5}{|l|}{ Metastasis (M) } \\
\hline Mo & $400(93.7 \%)$ & 198 (92.5\%) & $202(94.8 \%)$ & 0.123 \\
\hline MI & $3(0.7 \%)$ & $3(1.4 \%)$ & $0(0.0 \%)$ & \\
\hline Unknown & 24 (5.6\%) & $13(6.1 \%)$ & II (5.2\%) & \\
\hline
\end{tabular}

Abbreviations: AUC, area under curve; BCR, biochemical recurrence; DEirlncRNAs, differentially expressed irlncRNAs; HLA, human leukocyte antigen; ICB, immune checkpoint blockade; IPS, immunophenoscore; ir-genes, immune-related genes; irlncRNAs, immune-related long non-coding RNAs; mCRPC, metastatic castration-resistant prostate cancer; ROC, receiver operating characteristic; rPFS, radiographic progression-free survival; TCGA, the Cancer Genome Atlas; TMB, tumor mutation burden.

We found that the risk coefficient and the probability of BCR of the high-risk group were higher than those of the low-risk group. The higher risk score meant that prostate cancer patients were more likely to have BCR and owned shorter BCR-free time. Then, the heatmap was to display the expression profiles of the 10 DEirlncRNAs pairs in the training set (Figure 3C). Kaplan-Meier survival curve showed that the BCR-free time of the high-risk group was significantly lower than that of the low-risk group (Figure 3D). Furthermore, to illustrate the sensitivity and specificity of the risk score in the training set, the timedependent receiver operating characteristic (ROC) analysis was conducted. The area under the curves (AUCs) of the signature constructed by 10 DEirlncRNAs pairs in 1, 3, and 5 years were $0.821,0.800$, and 0.810 , respectively (Figure 3E). The hazard ratio (HR) of risk score and
95\% CI were 8.102 and 3.536-18.562 in univariate Cox regression analysis $(\mathrm{P}<0.001)$ (Figure $3 \mathrm{~F})$. In addition, a multivariate Cox regression analysis was conducted and revealed that the signature $(\mathrm{HR}=6.250,95 \% \mathrm{CI}=2.392$ 16.329, $\mathrm{P}<0.001)$ was of vital significance for BCR prediction (Figure $3 \mathrm{G}$ ). Using univariate and multivariate Cox regression analyses, we found that the signature was an independent prognostic factor in the training set. The detailed values of univariate and multivariate Cox regression analyses in the training set were recorded in Table S2.

\section{Validation of the Signature in the Testing Set and the Entire Set}

In order to further verify the predictive ability and stability of the signature in predicting the BCR of prostate cancer patients, we validated it in the testing set and the entire 
A

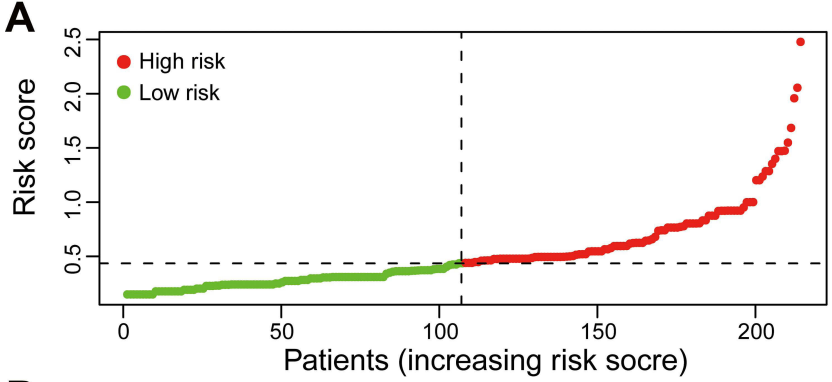

B

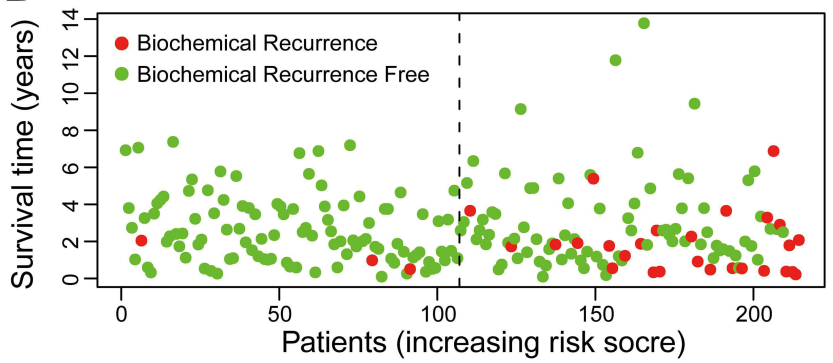

C

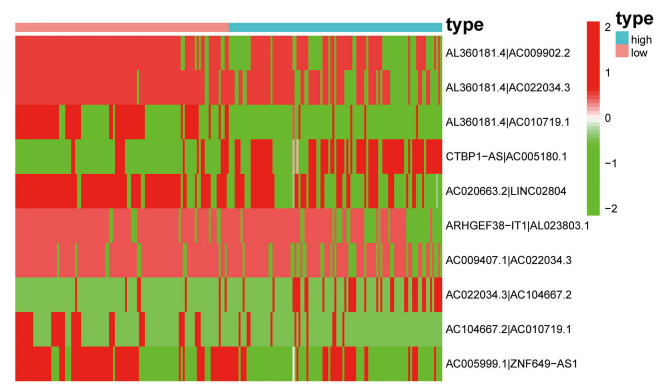

D

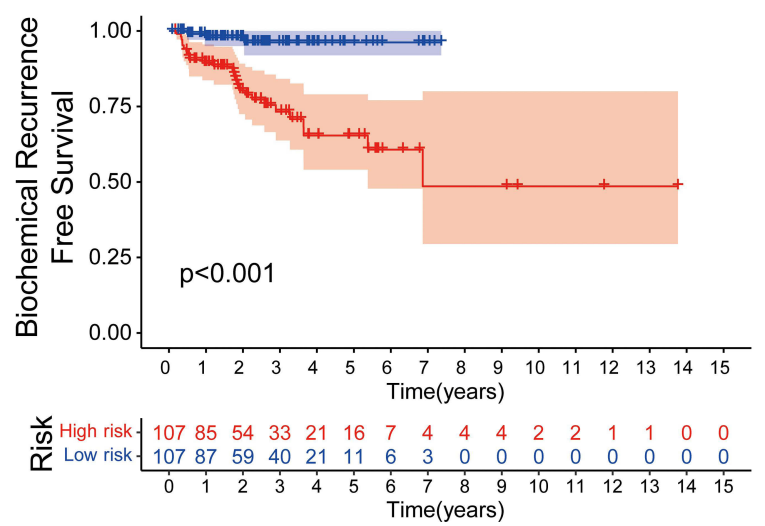

E

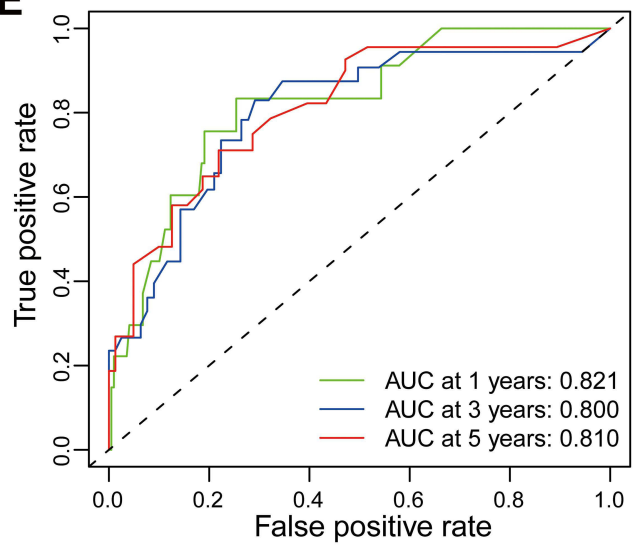

$\mathbf{F}$

$p$ value Hazard ratio

$0.335 \quad 1.032(0.968-1.102)$

Age

Grade

$0.006 \quad 1.635(1.152-2.320)$

$0.0101 .474(1.096-1.981)$

Riskscore $<0.0018 .102(3.536-18.562)$

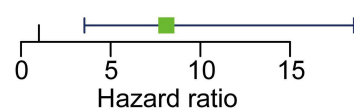

G

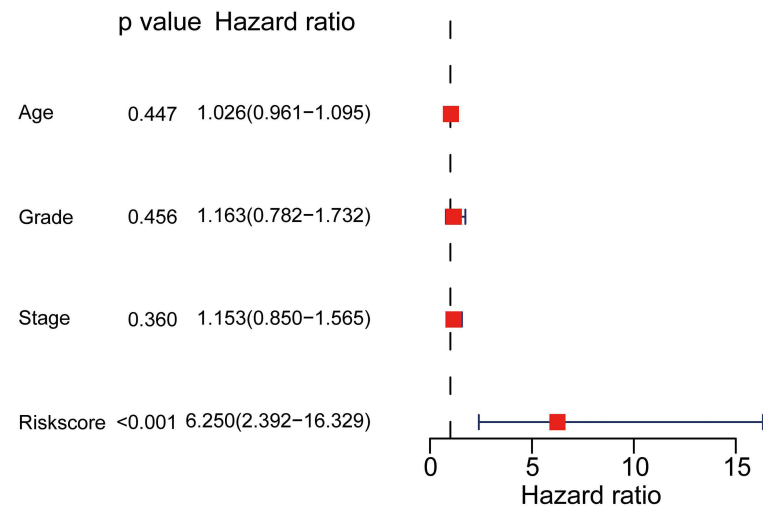

Figure 3 Evaluation of a risk model for prostate cancer in the training set. (A) The risk curve of each sample reordered by risk score. (B) The scatter plot of the samples of BCR. (C) Heatmap showed the expression profiles of the signature in the low-risk group and high-risk group. (D) Biochemical recurrence analysis for the signature. (E) Time-dependent ROC analysis curve for the signature. (F) Forest plot for univariate Cox regression analysis. (G) Forest plot for multivariate Cox regression analysis.

TCGA set. The score distribution and survival status diagram showed a significant difference in BCR between the low- and high-risk groups, with a gradual increase in BCR as the risk score increased (Figure $4 \mathrm{~A}$ and $\mathrm{B}$ and Figure $\underline{\mathrm{S} 2 \mathrm{~A}}$ and $\mathrm{B})$. The expression profiles of the 10 DEirlncRNAs pairs in the testing set and the entire set were depicted in the heatmap (Figures 4C and $\underline{\mathrm{S} 2 \mathrm{C}}$ ). Kaplan-Meier survival analysis of the testing set and the entire set showed no difference with the results of the training set: patients in the high-risk group had a worse BCR than those in the low-risk group (Figures 4D and $\underline{\mathrm{S} 2 \mathrm{D}})$. Consisting with the result in the training set, the 1-, 
A

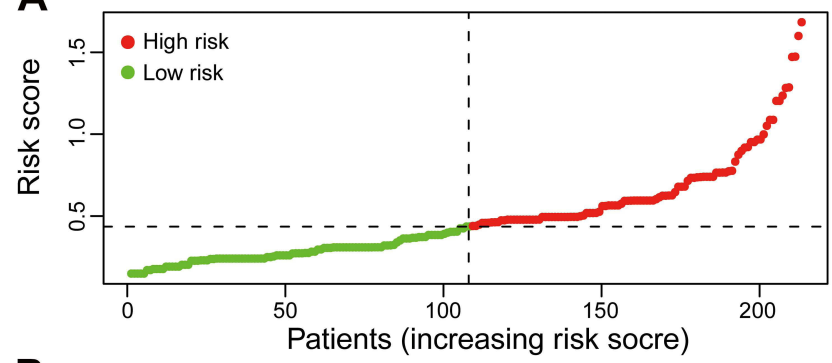

B

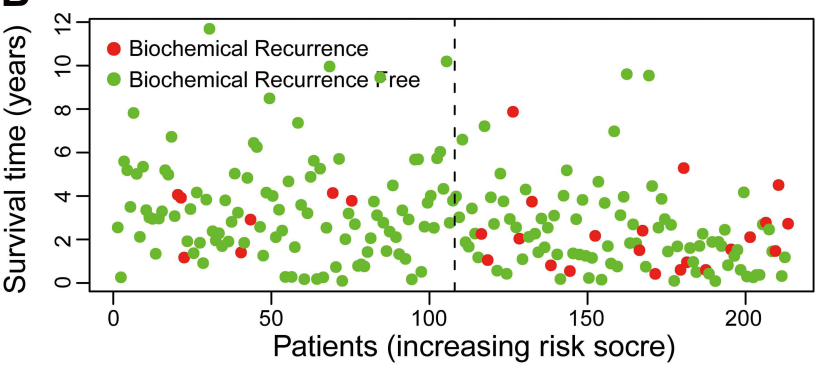

C

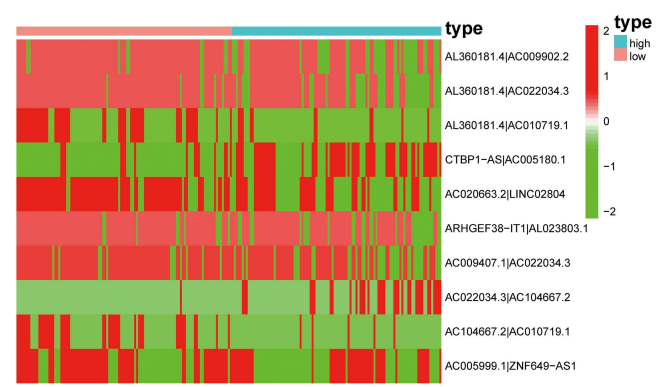

D

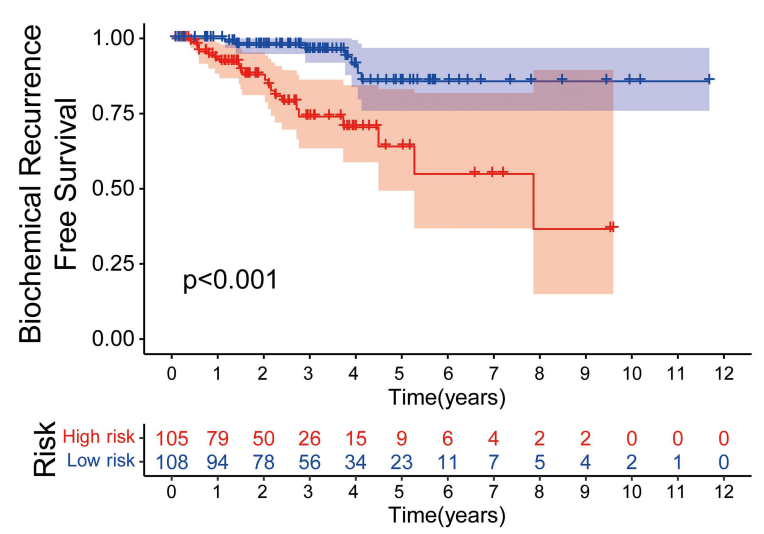

E

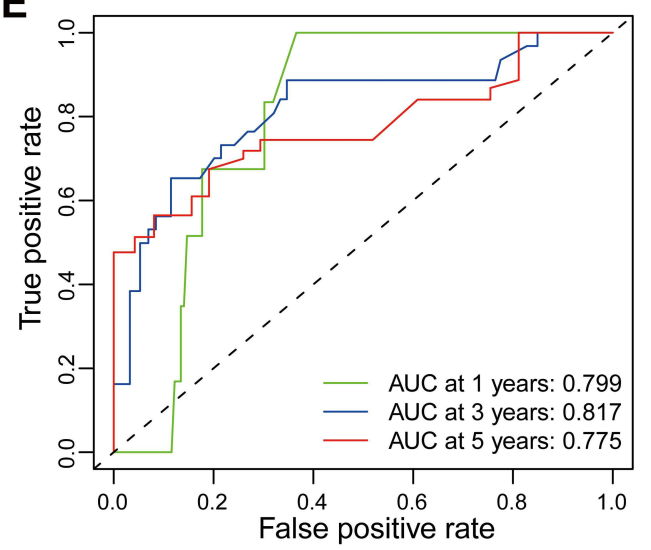

$\mathbf{F}$ p value Hazard ratio ।

Age

$0.7110 .989(0.932-1.049) \quad$ 甲

Grade $\quad<0.001 \quad 1.879(1.313-2.688) \quad$ I

Stage $\quad 0.010 \quad 1.544(1.107-2.152)$

Riskscore $<0.001$ 8.964(3.228-24.889)

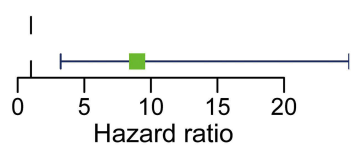

G

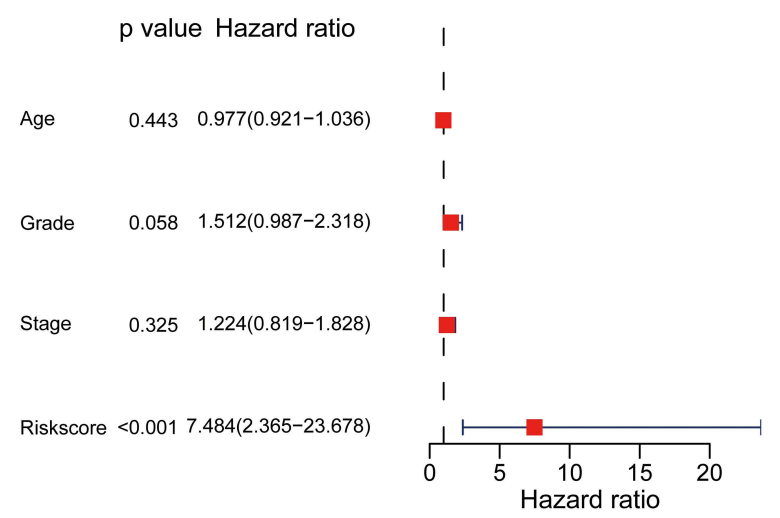

Figure 4 Evaluation of a risk model for prostate cancer in the testing set. (A) The risk curve of each sample reordered by risk score. (B) The scatter plot of the samples of BCR. (C) Heatmap showed the expression profiles of the signature in the low-risk group and high-risk group. (D) Biochemical recurrence analysis for the signature. (E) Time-dependent ROC analysis curve for the signature. (F) Forest plot for univariate Cox regression analysis. (G) Forest plot for multivariate Cox regression analysis.

3-, and 5-years AUC of the signature in the testing set was $0.799,0.817$, and 0.775 , respectively (Figure $4 \mathrm{E}$ ). In the end, we confirmed that the signature could act as a robust and independent prognostic indicator for prostate cancer patients by performing univariate and multivariate regression analyses in the testing set and the entire set (Figure 4F and G and Figure S2E and F). Tables S3 and S4 documented detailed values of univariate and multivariate Cox regression analyses in the testing set and the entire set. 
A
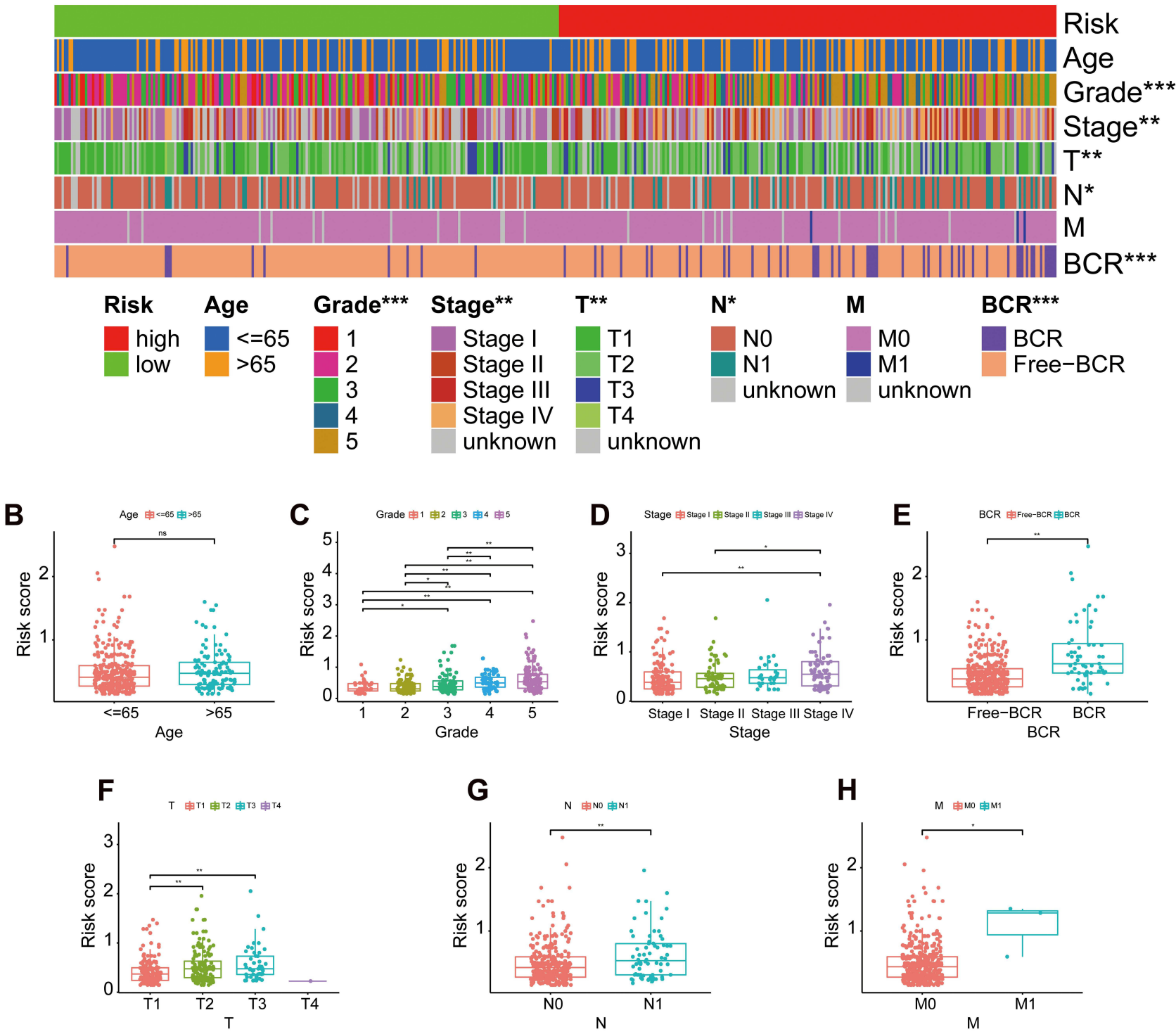

Figure 5 The relationship between the signature and different clinical features. A strip chart $(\mathbf{A})$ along with the scatter diagram showed the relationship among $(\mathbf{B})$ age, $(\mathbf{C})$ tumor grade, (D) stage, (E) BCR, (F) T stage, $(\mathbf{G}) \mathrm{N}$ stage, $(\mathbf{H}) \mathrm{M}$ stage and the risk score. . $* \mathrm{P}<0.05$, $* * \mathrm{P}<0.01$, and $* * * \mathrm{P}<0.00 \mathrm{I}$.

\section{Correlation Between Clinical Characteristics and the Signature}

The signature served as a good predictor of BCR in prostate cancer, and its relationship with clinical characteristics needed further exploration. The strip chart (Figure 5A) was applied to reveal that there are significant differences between low- and high-risk groups in grade $(\mathrm{P}<0.001)$, stage $(\mathrm{P}<0.01)$, T stage $(\mathrm{P}<0.01)$, $\mathrm{N}$ stage $(\mathrm{P}<0.05)$, and $\mathrm{BCR}(\mathrm{P}<0.001)$. Furthermore, although our signature was not associated with age, it was significantly related to the clinical characteristics, including grade, stage, $\mathrm{BCR}$, $\mathrm{T}$ stage, $\mathrm{M}$ stage, and $\mathrm{N}$ stage
(Figure 5B-H), suggesting it might play a crucial role in prostate cancer progression.

In order to further verify the prognostic value and explore the broad applicability of the signature, we conducted a survival analysis through stratification analysis between the low- and high-risk groups in the entire cohort (Figure 6A-H). According to the subgroups divided by age, tumor grade, and stage, the BCR-free time of the lowrisk group was longer than that of the high-risk group. In conclusion, these results suggested that the signature might play a key role in the oncogenesis and progression of 

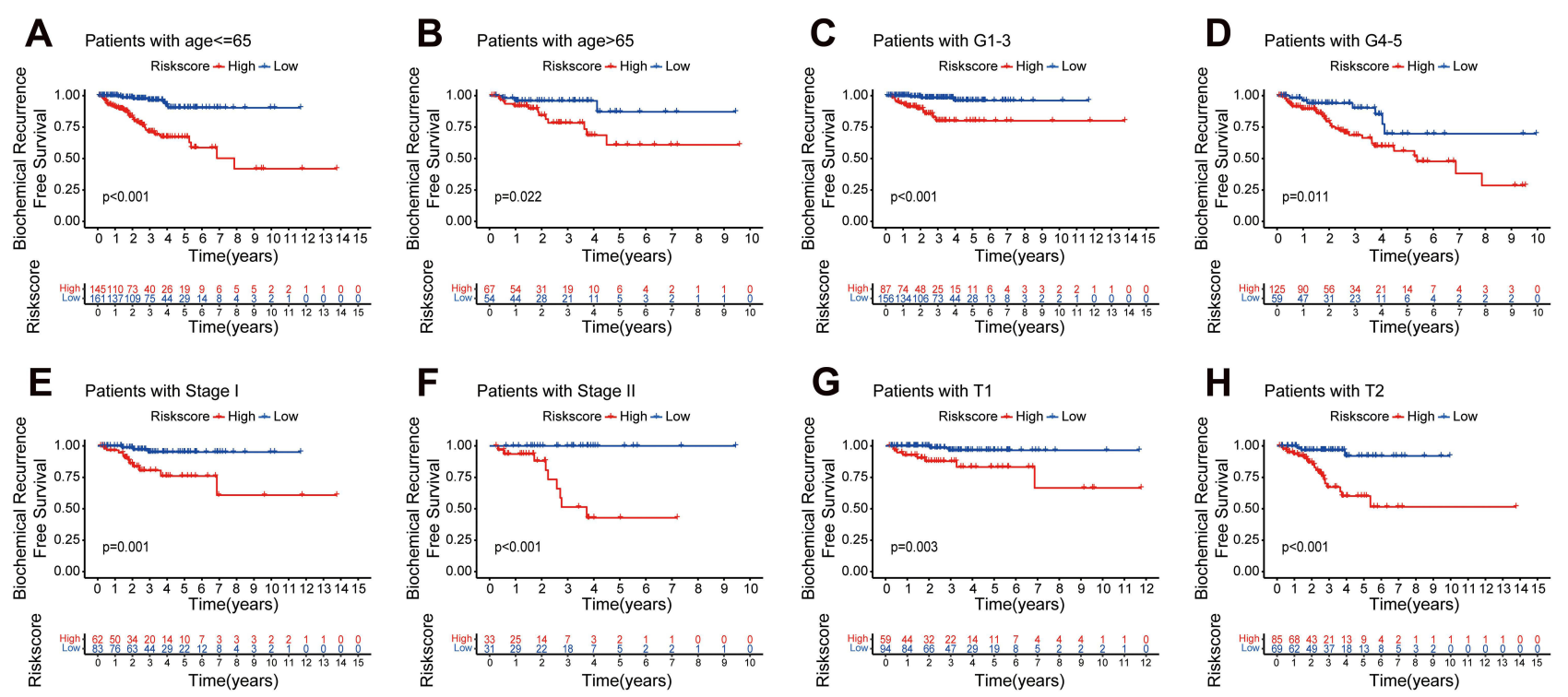

Figure 6 Stratification survival analyses. (A-H) Kaplan-Meier curve analyses of BCR-free time in subgroups stratified by different clinical features.

prostate cancer and effectively determine the prognosis of prostate cancer.

\section{Construction and Validation of the Prognostic Nomogram Based on the Signature}

To assess the accuracy of the signature in the entire set, the AUC for the signature prognostic model $(\mathrm{AUC}=0.815)$ was calculated and compared with the AUC of clinical factors (AUC of age $=0.585$, AUC of grade $=0.647$, AUC of stage $=0.724$ ), which confirmed that the signature was exceptionally reliable and better than the existing clinical characteristics (Figure 7A). Similarly, DCA was carried out in the TCGA entire set and suggested that the signature had excellent net benefits, which proved that the signature has better predictive accuracy than traditional clinical factors (Figure 7B). Next, we constructed a nomogram to predict the 1-, 2-, and 3-year incidence of $\mathrm{BCR}$ in prostate cancer patients based on risk score and clinical characteristics (Figure 7C). Calibration plots showed ideal agreement between observed and predicted rates for predicting 1-, 2-, and 3-year BCR-free time for prostate cancer patients (Figure 7D-F). Thus, these results suggested that the signature-based nomogram with good prognostic ability could be used in clinical practice.

\section{Somatic Mutations in Two Risk Groups of Prostate Cancer}

The somatic mutation profiles of prostate cancer patients from the TCGA database were analyzed to provide a comprehensive landscape of mutation profiles in the lowand high-risk groups. The waterfall plot showed the mutation information of the top 20 genes with the highest alteration frequency in the two groups (Figure 8A and B). It revealed that $42.23 \%$ of the 206 patients in the low-risk group occurred somatic mutations, while $64.36 \%$ of the 202 patients in the high-risk group had somatic mutations. Furthermore, after a detailed ranking of the different mutation types, we found that missense mutations and single nucleotide polymorphism (SNP) were the most primary mutational types in both groups. Besides, C > $\mathrm{T}$ transversion accounted for the most predominant of the single nucleotide variants (Figure $8 \mathrm{C}$ and D). Co-expression analysis of these mutant genes was conducted, which represented the coincident and exclusive relationship between the mutant genes (Figure 8E and F). Then, we proceeded to analyze the relationship between the signature and the TMB score. The results show that the TMB in the high-risk group is higher than in the low-risk group (Figure 9A), and the Pearson correlation analysis corroborated that TMB was positively correlated with the risk score (Figure 9B). TP53 mutant is one of the most common mutant genes in prostate cancer and can serve as a prognostic biomarker of prostate cancer. In order to understand whether our signature predicting BCR results was better than TP53 mutation status, we defined TP53 wild and TP53 mutant in the low-risk group and high-risk group as TP53 wild/low, TP53 wild/high, TP53 mutant/low, and TP53 mutation/high, respectively. First of all, patients with TP53 mutation had shorter BCR-free time 

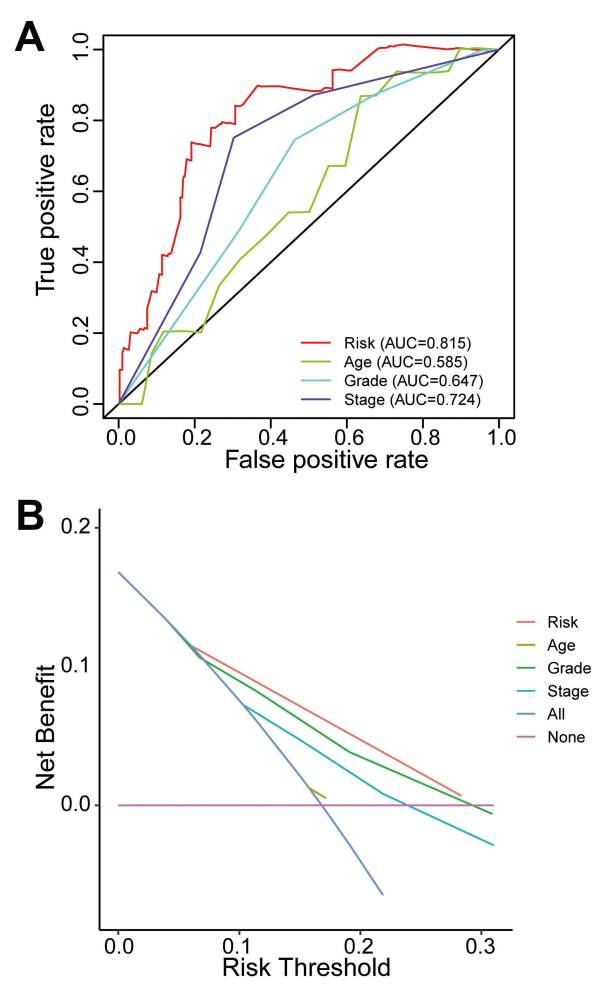

D

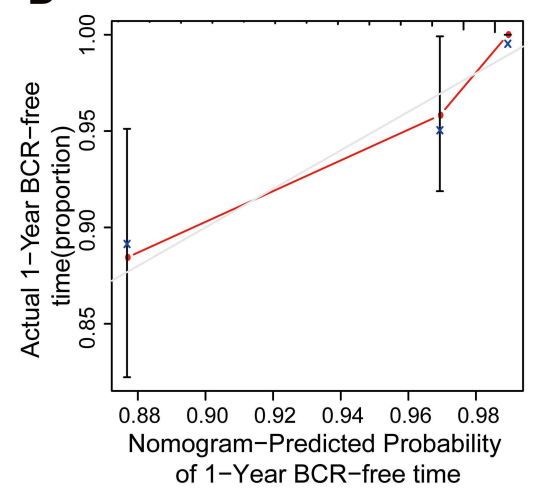

C

Points

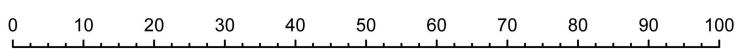

Age

Grade

Stage

Riskscore

Total Points

ताrrmाr
$80 \quad 55$
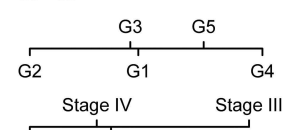

Stage I Stage II

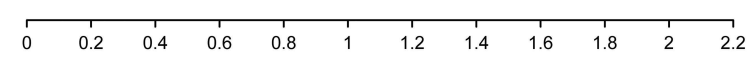

1-year BCR-free survival

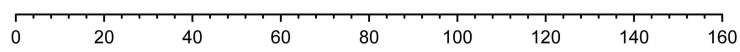

1-year BCR-free survival

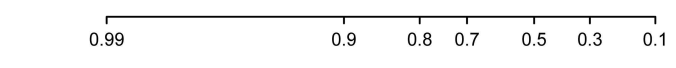

2-year BCR-free survival

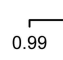

3-year BCR-free survival

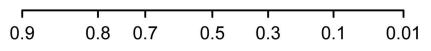

E

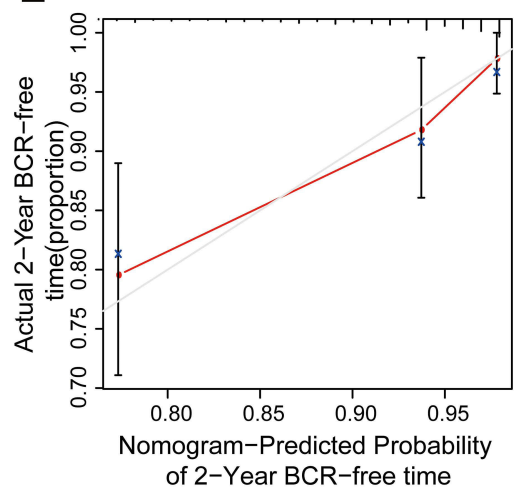

$\mathbf{F}$

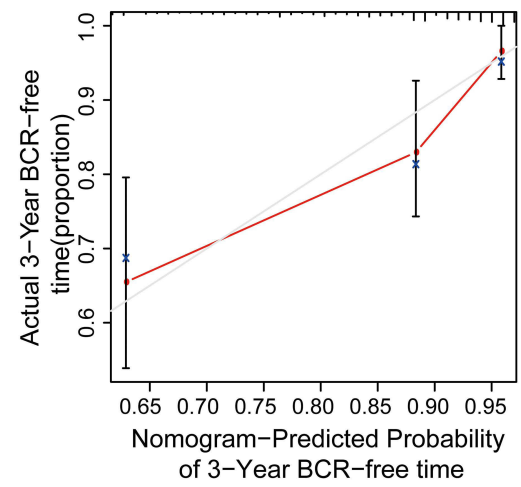

Figure 7 Construction and validation of nomogram. (A) Time-dependent ROC analysis curve for the signature and clinical factors in the entire set. (B) Decision curve analysis of the signature and different clinical factors. (C) The nomogram predicted the probability of the I-, 2-, and 3-year BCR-free time. (D-F) Calibration plot for the validation of the nomogram.

than patients with TP53 wild (Figure 9C). Second, patients with TP53 mutation/high presented the worst BCR (Figure 9D). Unexpectedly, patients with TP53 wild/high showed worse BCR than patients with TP53 mutation/low, which indicated that our signature could predict the BCR of prostate cancer more effectively and was superior to TP53 mutation status.

\section{Immune Status Analysis in Two Risk Groups for Prostate Cancer}

The signature was strongly associated with the immune status of prostate cancer, and different immune status was also differently expressed in low- and high-risk groups. Based on this result, CIBERSORT and TIMER data were further used to analyze the correlation between the signature and immune cell subtype infiltration. Spearman correlation analysis was performed, and the results showed that our signature was closely related to multiple immune cells (Figure 10A). In addition, the different algorithms of immune responses were depicted in the heatmap (Figure 10B). The low-risk group exhibited significantly higher infiltrated abundance of $\mathrm{CD} 4+\mathrm{T}$ cells, CD8+ T cells, neutrophils, macrophage, myeloid dendritic cells, $\mathrm{B}$ naive cells, and B cells compared to the low-risk group 

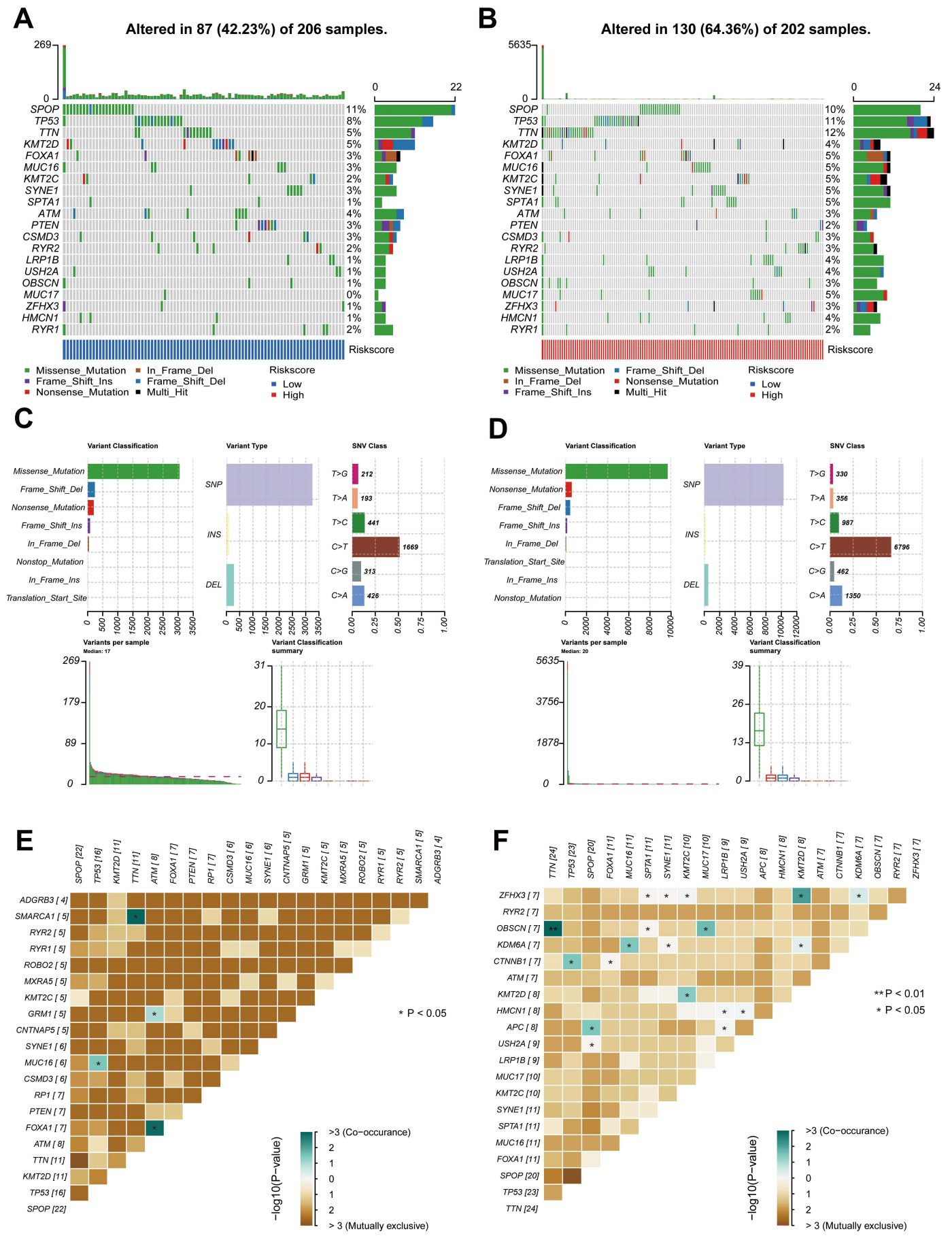

Figure 8 Landscape of mutation profiles between low- and high-risk groups. (A and B) Mutation information of the genes with high mutation frequencies in the low- and high-risk groups. (C and D) Variant classification, variant type, SNV classification, variants in per sample, and summary of variant classification in the two groups. (E and $\mathbf{F}$ ) Co-expression analysis of Top 20 mutant genes in the two groups. $* \mathrm{P}<0.05$ and $* * \mathrm{P}<0.01$.

$(\mathrm{P}<0.01$, Figure 10C-I). However, the low-risk group was more negatively associated with $\mathrm{B}$ memory cells $(\mathrm{P}<$ 0.01 , Figure $10 \mathrm{~J})$. As a representative of the tumor microenvironment, the ESTIMATE algorithm was applied to calculate the stromal score, immune score, ESTIMATE score, and tumor purity (Figure S3).
Subsequently, we used the ssGSEA algorithm to evaluate the immune cell infiltration of the low-risk and high-risk groups of prostate cancer (Figure 11A). Meanwhile, the result of immune function suggested that CCR, immune checkpoints, HLA, MHC class I, and Parainflammation showed significant differences (Figure 11B). Furthermore, the 
A

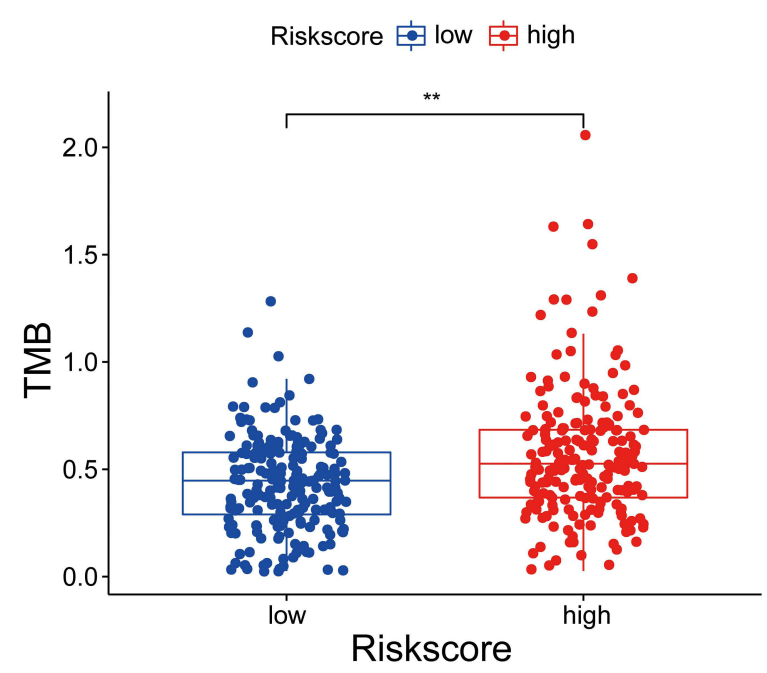

C

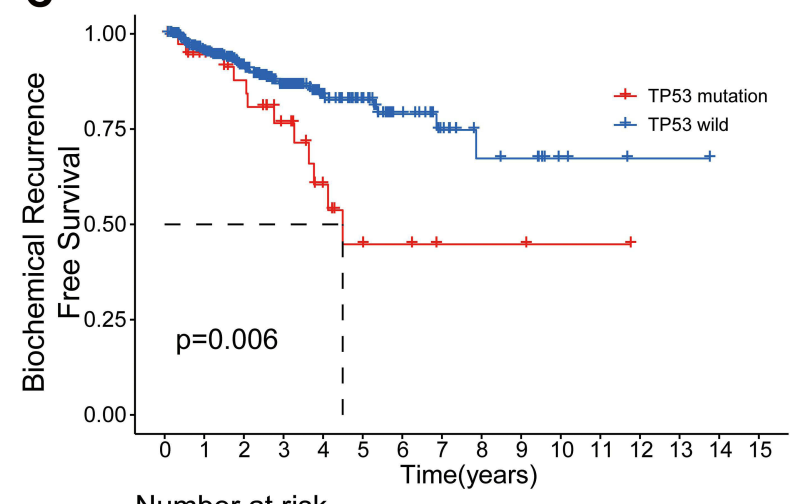

Number at risk

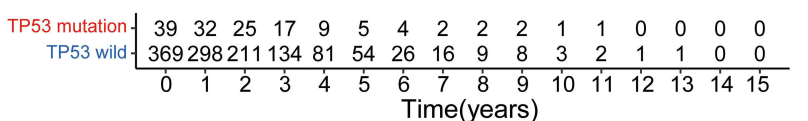

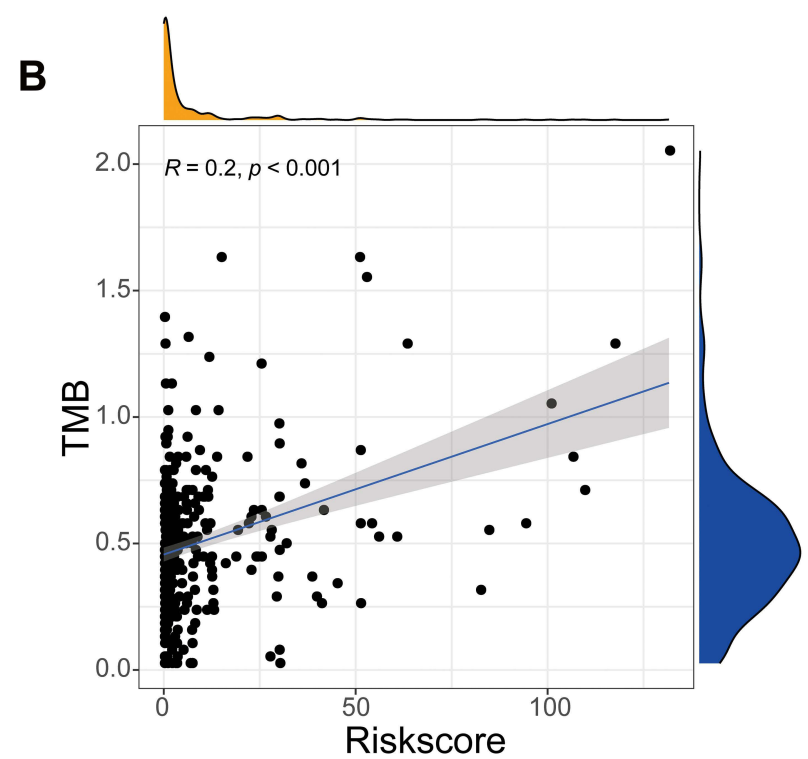

D

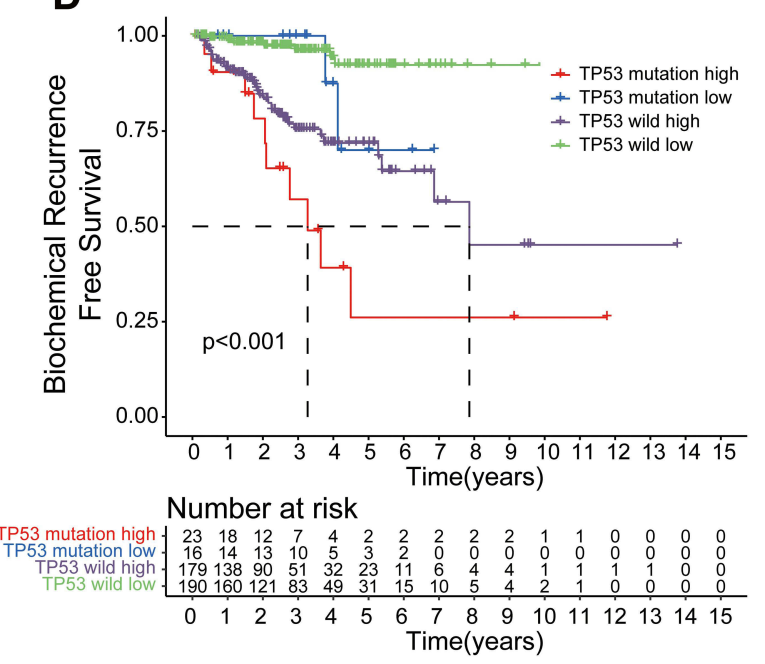

Figure 9 The relationship between TMB and the signature. (A) The level of TMB in the low- and high-risk groups. (B) Pearson correlation analysis between TMB and risk score. (C) Kaplan-Meier curve analysis of BCR-free time for patients with TP53 wild or TP53 mutation. (D) Kaplan-Meier curve analyses of BCR-free time for patients with different TP53 status and risk groups. $* * P<0.01$.

differential analysis of the HLA genes expression and immune checkpoint expression demonstrated distinctions in the immune status of the two groups as well as higher HLA gene expression in the low-risk group than in the high-risk group (Figure 11C and D). Finally, to evaluate the potential response of immune checkpoint blockade (ICB) for prostate cancer patients, we explored the relationship between IPS and our signature (Figure 11E). The IPS was higher in the lowrisk group, which appeared to have stronger immunogenicity.

\section{Correlation Analysis of Drug Treatment}

In clinical practice, chemotherapeutic and endocrine drugs were used to treat patients with prostate cancer. ${ }^{37,38}$ Using prostate cancer data from the TCGA project, we analyzed the relationship between the signature and the efficacy of common chemotherapy and endocrine drugs. We found that the endocrine drug of bicalutamide $(\mathrm{P}<0.01$, Figure 12A) the chemotherapeutic drug of Docetaxel (P $<0.01$, Figure 12B) had a lower semi-inhibition rate (IC50) in the low-risk group. However, the IC50 of Mitomycin $\mathrm{C}(\mathrm{P}<0.01$, Figure $12 \mathrm{C})$, Doxorubicin $(\mathrm{P}<$ 0.01 , Figure 12D), and Etoposide $(\mathrm{P}<0.01$, Figure 12E) were lower in the high-risk group. Olaparib was a new type of targeted drug that acted to block poly ADP ribose polymerase (PARP) protein. ${ }^{39}$ The high-risk group was more sensitive to Olaparib than the low-risk group $(\mathrm{P}<$ 


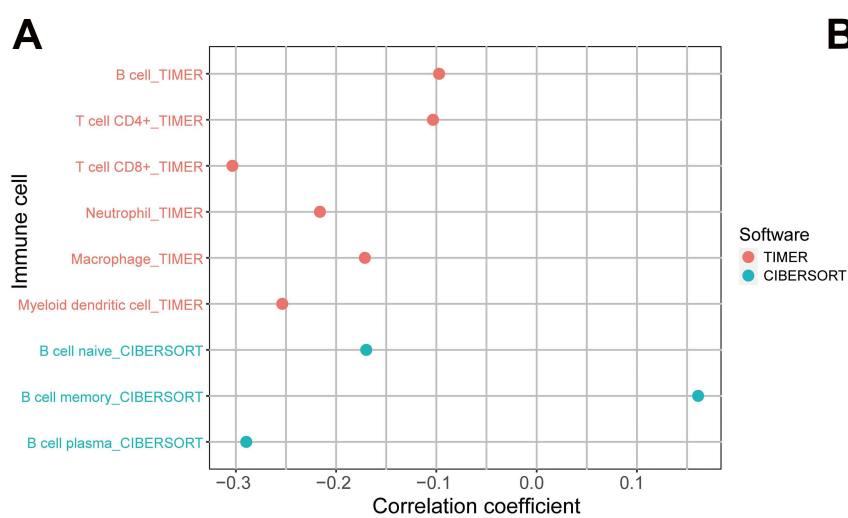

C Risk 官 low 官 high

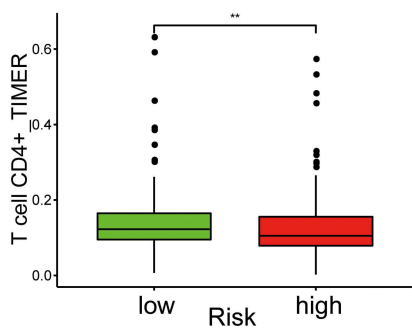

D Risk 审 low 审 high
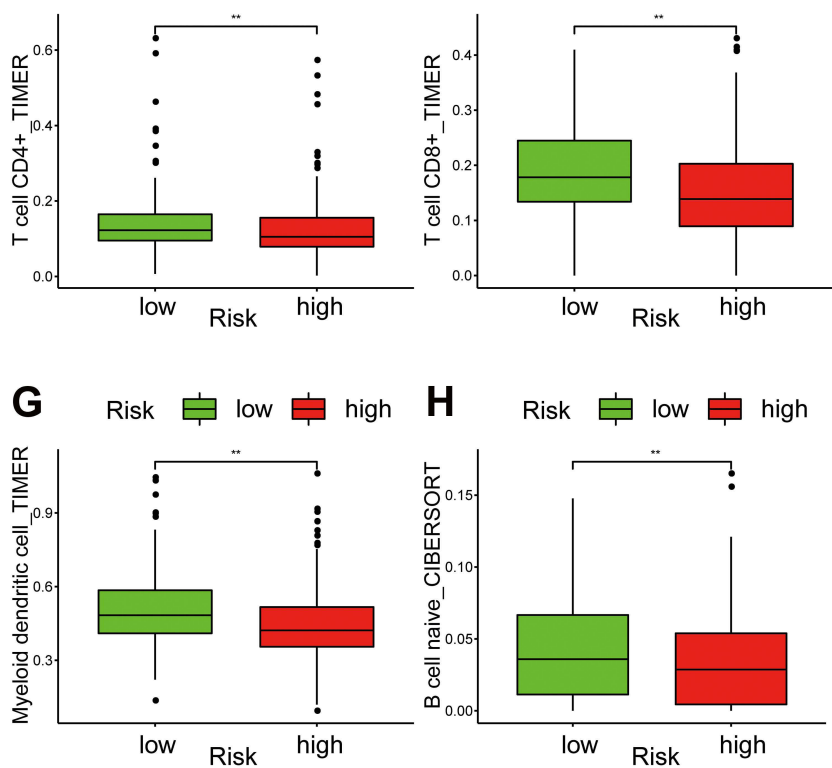

B
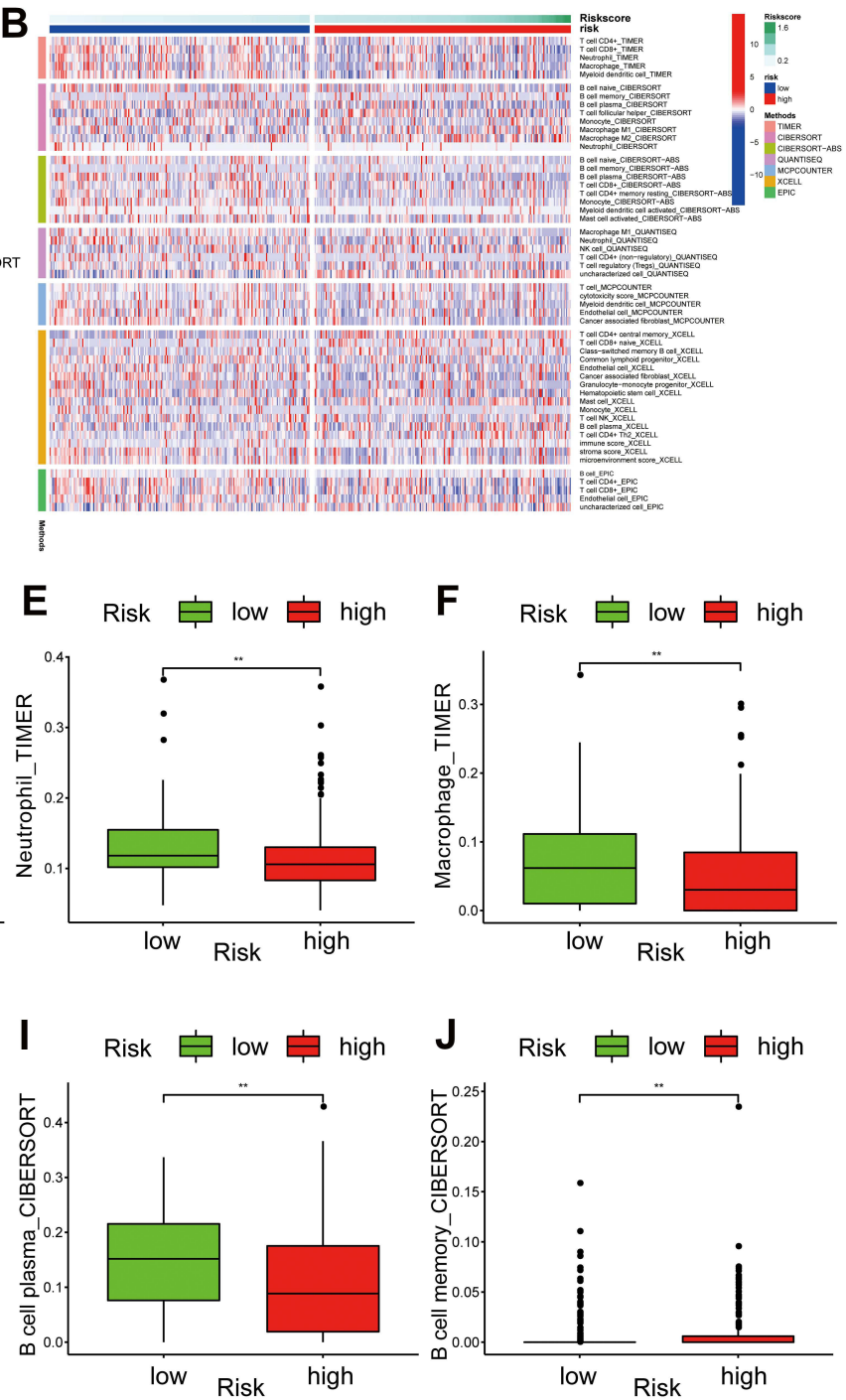

Figure 10 Tumor immune microenvironment between low- and high-risk groups. (A) The relationship between the signature and tumor-infiltrating immune cells. (B) Heatmap of abundance of immune cells in the low- and high-risk groups. (C-J) Correlation between the signature and the infiltration of immune cell subtypes: (C) CD4+ T cells, (D) CD8+ T cells, (E) neutrophils, (F) macrophage, (G) myeloid dendritic cells, (H) B naive cells, (I) B cells, and (J) B memory cells. **P< $0.0 \mathrm{I}$.

0.01, Figure 12F). Our results indicated that the signature could provide a basis for clinical selection of endocrine drugs, chemotherapy, and targeted drugs and offer patients more precise treatments.

\section{Discussion}

Prostate cancer develops in the prostate gland and is one of the most common malignant tumors in men. ${ }^{2,40}$ Early diagnosis and timely treatment are urgent measures to improve the curative effect of prostate cancer. Up to $40 \%$ of patients still had BCR after radical surgery. ${ }^{41}$ Due to the heterogeneity of tumors and the diversity of biomolecules, the existing methods for predicting the prognosis of prostate cancer remained inadequate. Therefore, in this research, we developed a novel signature to predict the prognosis of prostate cancer, determine the possibility of BCR, distinguish high-risk patients, and customize individual medication plans for them.

In recent years, with the in-depth exploration of the transcriptome, it has been discovered that about $4 / 5$ of the transcripts in the human genome are protein non-coding genes, including lncRNAs. ${ }^{42}$ The role of lncRNAs was involved in the occurrence, development, invasion, and metastasis of malignant tumors in many ways. ${ }^{43-45}$ Recent studies have focused on quantifying the expression level of transcripts and analyzing the signal characteristics of lncRNAs to assess the prognosis of patients with malignant tumors. ${ }^{46,47}$ However, in our research, for the first 
A

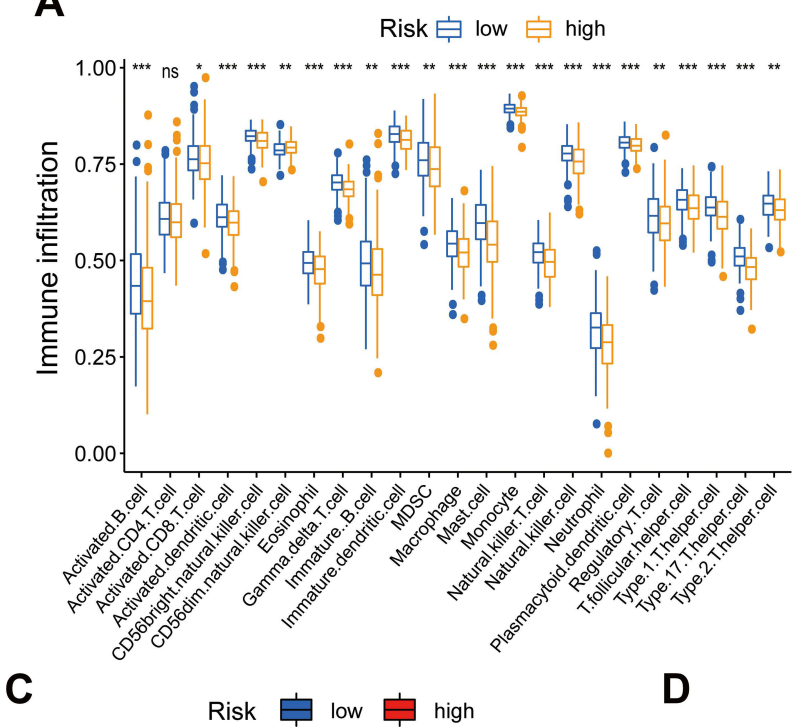

B
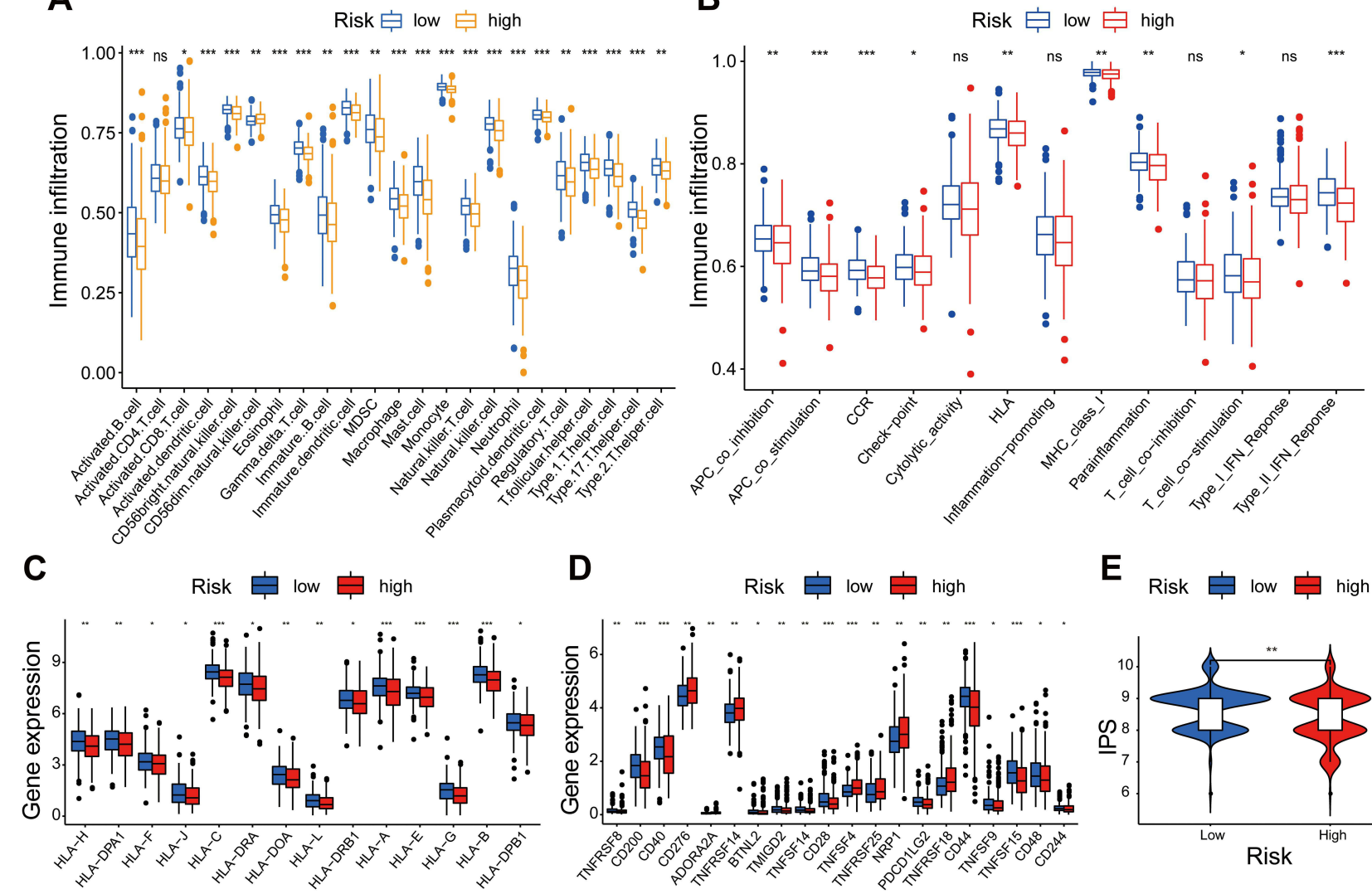
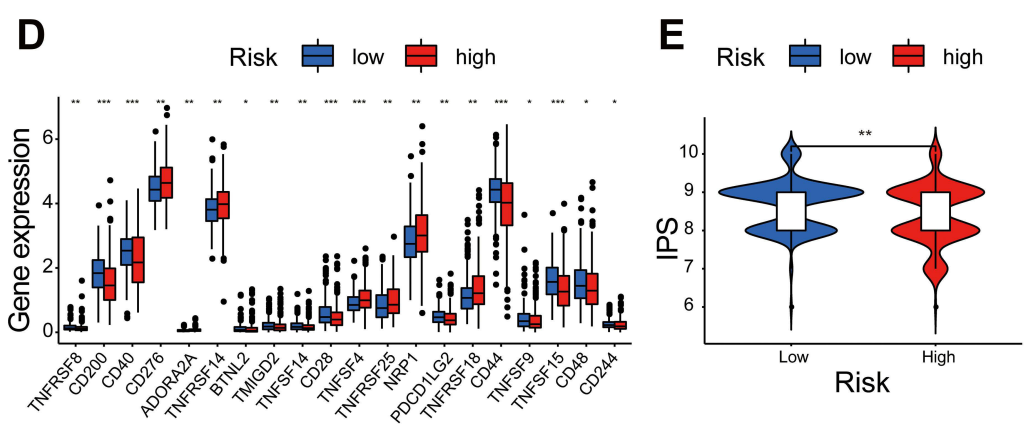

Figure I I Immune cell infiltration and immune function by ssGSEA algorithm. (A) The differences in the immune cells in the low- and high-risk groups. (B) Immune function in the two groups. (C) The expression of HLA family in the two groups. (D) The expression of immune checkpoint in the two groups. (E) The difference analysis of IPS between the two groups. ${ }^{*} \mathrm{P}<0.05$, $* * \mathrm{P}<0.01$, and $* * * \mathrm{P}<0.001$.

time, we constructed a signature based on the combination of two IncRNAs without knowing the abundance of IncRNAs, which could effectively predict the BCR of prostate cancer.

A large number of studies have highlighted the indispensable relationship between immunity and malignant tumors, which inspired us to consider prostate cancer from an immune perspective. ${ }^{48,49} \mathrm{BCR}$ is one of the main reasons for the death of prostate cancer patients. At present, many studies have shown that certain immunerelated gene and protein indicators can predict the BCR of prostate cancer. Shao et $\mathrm{al}^{50}$ constructed an immunerelated genes signature and identified the signatures that were associated with BCR of prostate cancer. Wang et $\mathrm{al}^{51}$ highlighted the prognostic value of serum immune-related proteins for predicting aggressiveness, $\mathrm{BCR}$, and progression of prostate cancer. Nevertheless, a growing number of studies reported that without a mission to encode proteins, IncRNAs were more specific than other types of markers in indicating the actual condition of the tumor, ${ }^{52}$ which was also in line with our results. In our study, the timedependent ROC curve analyses demonstrated that this signature in predicting BCR was more reliable than current clinical indicators. In addition, univariate and multivariate Cox regression analyses indicated that this signature might be superior to other clinicopathologic features and served as a better independent factor in predicting the BCR of prostate cancer. Besides, we constructed a nomogram and used the calibration plots to prove that the observed rate was consistent with the prediction rate in predicting the clinical application of BCR. Meanwhile, the results of DCA confirmed that the predictive power of our signature was better than traditional clinical features. In order to further verify the wide applicability of the signature, we conducted a stratified survival analysis of different clinical characteristics, which revealed that our signature could be equally applicable to different clinical stages. In summary, our signature could accurately and effectively predict BCR and provide new ideas for further improving the prognosis of prostate cancer patients. 

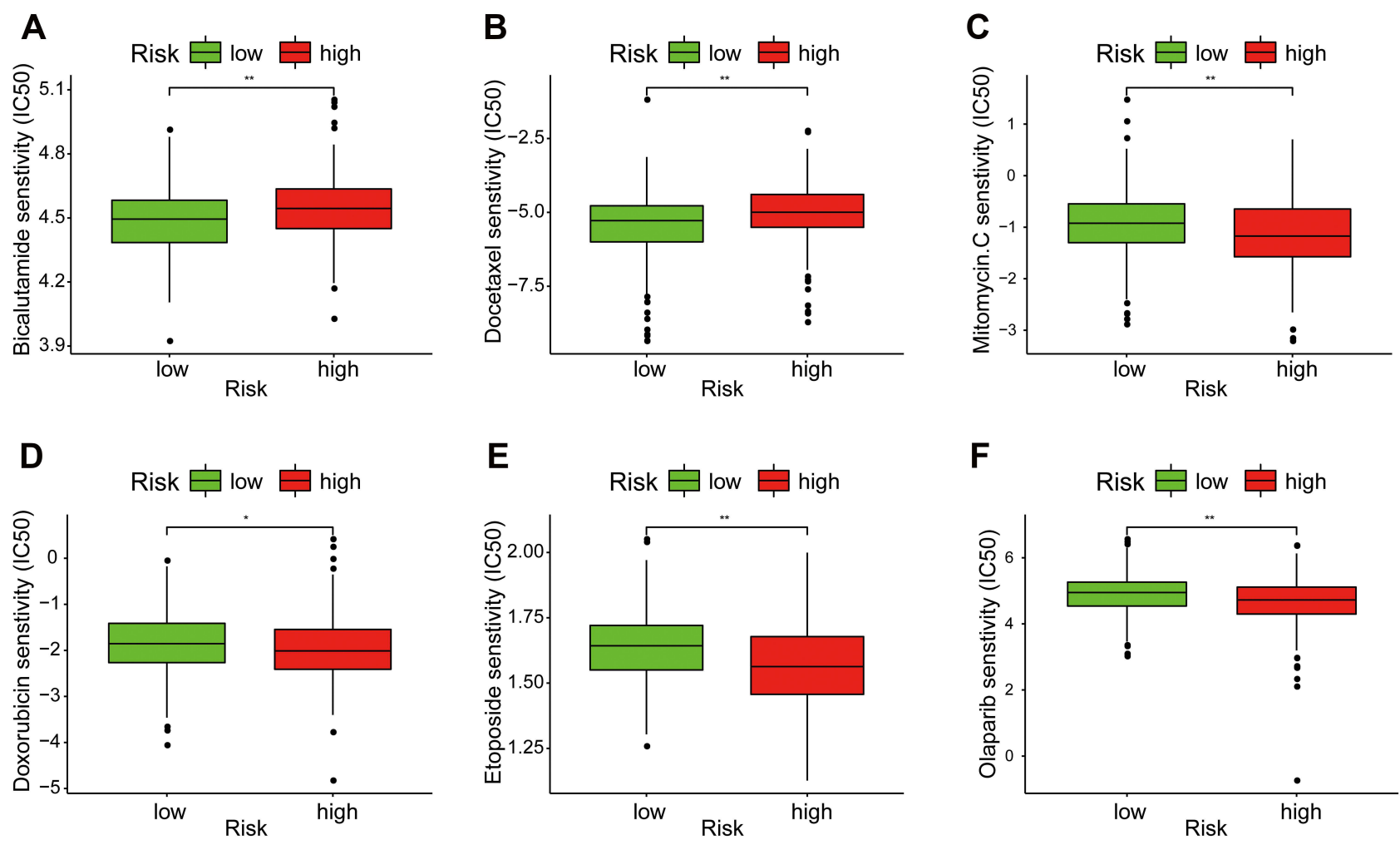

Figure 12 Assessment of medical treatment in low- and high-risk groups. (A-F) Correlation between the signature and the IC50 of the drugs: (A) Bicalutamide, (B) Docetaxel, (C) Mitomycin C, (D) Doxorubicin, (E) Etoposide, and (F) Olaparib. $* \mathrm{P}<0.05$ and $* * \mathrm{P}<0.01$.

In addition, we further analyzed the relationship between tumor-infiltrating immune cells and the signature to explore the tumor immune landscape. Weiner et $\mathrm{al}^{53}$ reported that an increase in plasma $\mathrm{B}$ was independently associated with prolonged recurrence-free survival of prostate cancer, which might improve the clinical prognosis by increasing interferon signal transduction, IgG expression, and Natural Killer (NK) cell activity. The immune activity and prognosis of prostate cancer were usually related to the existence and activity of CD8 + $\mathrm{T}$ cells in the tumor microenvironment. ${ }^{54} \mathrm{CD} 8+\mathrm{T}$ cells were the most active lymphocytes against tumors and had strong prognostic relevance in many solid tumors. ${ }^{55-57}$ Consistent with our results, the high-risk group had a higher probability of BCR while being negatively associated with plasma B cells and CD8+ T cells. Generally, prostate cancer is considered poorly immunogenic. ${ }^{58}$ To date, the ICB has yet to be FDA-approved for the management of prostate cancer, and the results of ICB clinical trials in prostate cancer have not been satisfactory. Phase III trials of CTLA-4 and PD-1 blockers in prostate cancer patients did not observe any difference in overall survival compared with placebo. ${ }^{59}$ However, only a minority of prostate cancer patients have shown particularly good results in immunotherapy, which might be because immunotherapy was tested mainly in unselected patients. ${ }^{60,61}$ Furthermore, we identified the correlation between the signature and immune functions such as HLA and immune checkpoints, providing new insights into immunotherapy. Analyzing the interaction between tumor cells and $\mathrm{T}$ cells or B cells would facilitate the development of new methods for tumor diagnosis and treatment. Perhaps, we could screen out specific prostate cancer patients to receive immunotherapy through the signature, which would be a new direction for prostate cancer immunotherapy in the future.

The total number of somatic coding mutations, termed as TMB, was associated with the emergence of new immune antigens that induced anti-tumor. ${ }^{62,63} \mathrm{TMB}$ is considered a predictive biomarker for cancer immunotherapy response and its relationship with melanoma, lung cancer, and urothelial cancer has been extensively studied ${ }^{64-67}$ Moreover, the results from a clinical trial in metastatic castration-resistant prostate cancer (mCRPC) patients taking the drug Olaparib showed that the radiographic progression-free survival (rPFS) of patients with 
BRCA or ATM mutations was more than twice that of patients without mutations (7.4 months vs 3.6 months). ${ }^{68}$ In our results, patients in the high-risk group had higher TMB levels. Also, the drug sensitivity results showed that the high-risk group was more sensitive to Olaparib than the low-risk group. This all suggested that the patients in the high-risk group were more likely to benefit from the treatment with Olaparib. In short, prostate cancer patients could get more precise treatments through our signature.

Endocrine therapy was the preferred treatment for early prostate cancer. ${ }^{69}$ Our results indicated that the low-risk group was more sensitive to the endocrine drug Bicalutamide. This would help clinicians pay more attention to the high-risk group to prevent premature progression of CRPC. Chemotherapy was closely related to immune cell infiltration. Studies have shown that B cells are related to the chemotherapy resistance of prostate cancer. ${ }^{70}$ NK cells could enhance the immunogenic cell death induced by chemotherapeutics. $^{71}$ The potential synergy between chemotherapy and immune cell has been well documented, although the exact mechanism remained to be studied. ${ }^{72,73}$ Docetaxel is a common chemotherapeutic drug for prolonging the life of metastatic CRPC patients. ${ }^{74}$ However, there is currently no selective choice of appropriate chemotherapy drugs for specific patients in clinical practice. Surprisingly, our results showed that the low- and high-risk groups had different sensitivities to different chemotherapy drugs. The low-risk group was more sensitive to Docetaxel, but the highrisk group was more inclined to Mitomycin C, Doxorubicin, and Etoposide. This meant that patients, who could be divided into specific groups by this signature, received more suitable chemotherapy drugs. Incorporation of our signature into chemotherapy sensitivity classification might further stratify patients with a better prognosis. This not only facilitated the choice of clinical medication for physicians but was also a boon to patients.

\section{Conclusions}

In summary, we have done a comprehensive evaluation on the ability of the signature to predict the BCR and its role in the immune landscape of prostate cancer. Our results provided a new approach toward new immunological research and treatment strategies for prostate cancer patients.

\section{Data Sharing Statement}

All data generated or analyzed during this study are included in this published article and the Supplement Material.

\section{Ethics Approval and Consent to Participate}

Not applicable. Since TCGA belongs to public database, the patients involved in the database have obtained ethical approval. Users can download relevant data for free for research and publish relevant articles. Our research is based on open-source data. The Second People's Hospital of Foshan clearly requires that the use of public data for research does not need to be submitted to the ethics committee for review, so there are no ethical issues and other conflicts of interest.

\section{Acknowledgments}

We would like to acknowledge the TCGA database.

\section{Funding}

No funding was received for conducting this study.

\section{Disclosure}

The authors report no conflicts of interest in this work.

\section{References}

1. Sung H, Ferlay J, Siegel RL, et al. Global cancer statistics 2020: GLOBOCAN estimates of incidence and mortality worldwide for 36 cancers in 185 countries. CA Cancer J Clin. 2021;71(3):209-249. doi: $10.3322 /$ caac. 21660

2. Siegel RL, Miller KD, Fuchs HE, Jemal A. Cancer statistics, 2021. CA Cancer J Clin. 2021;71(1):7-33. doi:10.3322/caac.21654

3. Mccormick B, Mahmoud A, Williams S, Davis J. Biochemical recurrence after radical prostatectomy: current status of its use as a treatment endpoint and early management strategies. Indian J Urol. 2019;35(1):6-17. doi:10.4103/iju.IJU_355_18

4. Carroll PH, Mohler JL. NCCN guidelines updates: prostate cancer and prostate cancer early detection. J Natl Compr Canc Netw. 2018;16(5S):620-623. doi:10.6004/jncen.2018.0036

5. Montironi R, Mazzucchelli R, Scarpelli M, et al. Prostate carcinoma II: prognostic factors in prostate needle biopsies. BJU Int. 2006;97 (3):492-497. doi:10.1111/j.1464-410X.2006.05973.x

6. Kreuz M, Otto DJ, Fuessel S, et al. ProstaTrend-a multivariable prognostic RNA expression score for aggressive prostate cancer. Eur Urol. 2020;78(3):452-459. doi:10.1016/j.eururo.2020.06.001

7. Joyce JA, Fearon DT. T cell exclusion, immune privilege, and the tumor microenvironment. Science. 2015;348(6230):74-80. doi:10.1126/science.aaa6204

8. Jhunjhunwala S, Hammer C, Delamarre L. Antigen presentation in cancer: insights into tumour immunogenicity and immune evasion. Nat Rev Cancer. 2021;21(5):298-312. doi:10.1038/s41568-02100339-z

9. Schaafsma E, Fugle CM, Wang X, Cheng C. Pan-cancer association of HLA gene expression with cancer prognosis and immunotherapy efficacy. Br J Cancer. 2021;125(3):422-432. doi:10.1038/s41416021-01400-2

10. Wickenhauser C, Bethmann D, Kappler M, et al. Tumor microenvironment, HLA class I and APM expression in HPV-negative oral squamous cell carcinoma. Cancers. 2021;13(4):620. doi:10.3390/ cancers 13040620 
11. Ladanyi A, Timar J. Immunologic and immunogenomic aspects of tumor progression. Semin Cancer Biol. 2020;60:249-261. doi:10.1016/j.semcancer.2019.08.011

12. Fridman WH, Zitvogel L, Sautes-Fridman C, Kroemer G. The immune contexture in cancer prognosis and treatment. Nat Rev Clin Oncol. 2017;14(12):717-734. doi:10.1038/nrclinonc.2017.101

13. Galluzzi L, Buque A, Kepp O, Zitvogel L, Kroemer G. Immunological effects of conventional chemotherapy and targeted anticancer agents. Cancer Cell. 2015;28(6):690-714. doi:10.1016/j. ccell.2015.10.012

14. Cech TR, Steitz JA. The noncoding RNA revolution-trashing old rules to forge new ones. Cell. 2014;157(1):77-94. doi:10.1016/j. cell.2014.03.008

15. Ulitsky I, Bartel DP. LincRNAs: genomics, evolution, and mechanisms. Cell. 2013;154(1):26-46. doi:10.1016/j.cell.2013.06.020

16. Cai $\mathrm{P}$, Otten $\mathrm{ABC}$, Cheng $\mathrm{B}$, et al. A genome-wide long noncoding RNA CRISPRi screen identifies PRANCR as a novel regulator of epidermal homeostasis. Genome Res. 2020;30(1):22-34. doi:10.1101/ gr.251561.119

17. Marchese FP, Raimondi I, Huarte M. The multidimensional mechanisms of long noncoding RNA function. Genome Biol. 2017;18 (1):206. doi:10.1186/s13059-017-1348-2

18. Lee S, Kopp F, Chang T, et al. Noncoding RNA NORAD regulates genomic stability by sequestering PUMILIO proteins. Cell. 2016;164 (1-2):69-80. doi:10.1016/j.cell.2015.12.017

19. Li Z, Zhang J, Liu X, et al. The LINC01138 drives malignancies via activating arginine methyltransferase 5 in hepatocellular carcinoma. Nat Commun. 2018;9(1):1572. doi:10.1038/s41467-018-04006-0

20. Martínez-Terroba E, Dimitrova N. Long noncoding RNA amplified in lung cancer rewires cancer pathways. J Cell Biol. 2020;219(9). doi: $10.1083 /$ jcb. 202007098

21. Schmitt AM, Chang HY. Long noncoding RNAs in cancer pathways. Cancer Cell. 2016;29(4):452-463. doi:10.1016/j.ccell.2016.03.010

22. Vallino L, Ferraresi A, Vidoni C, et al. Modulation of non-coding RNAs by resveratrol in ovarian cancer cells: in silico analysis and literature review of the anti-cancer pathways involved. $J$ Tradit Complement Med. 2020;10(3):217-229. doi:10.1016/j. jtcme.2020.02.006

23. Chen YG, Satpathy AT, Chang HY. Gene regulation in the immune system by long noncoding RNAs. Nat Immunol. 2017;18(9):962-972. doi: $10.1038 /$ ni.3771

24. Atianand MK, Caffrey DR, Fitzgerald KA. Immunobiology of long noncoding RNAs. Annu Rev Immunol. 2017;35(1):177-198. doi:10.1146/annurev-immunol-041015-055459

25. Wang L, Felts SJ, Van Keulen VP, et al. Integrative genome-wide analysis of long noncoding RNAs in diverse immune cell types of melanoma patients. Cancer Res. 2018;78(15):4411-4423. doi:10.1158/0008-5472.CAN-18-0529

26. de Lima DS, Cardozo LE, Maracaja-Coutinho V, et al. Long noncoding RNAs are involved in multiple immunological pathways in response to vaccination. Proc Natl Acad Sci UsA. 2019;116 (34):17121-17126. doi:10.1073/pnas.1822046116

27. Yu AD, Wang Z, Morris KV. Long noncoding RNAs: a potent source of regulation in immunity and disease. Immunol Cell Biol. 2015;93 (3):277-283. doi:10.1038/icb.2015.2

28. Sun Z, Jing C, Xiao C, Li T. Long non-coding RNA profile study identifies an immune-related IncRNA prognostic signature for kidney renal clear cell carcinoma. Front Oncol. 2020;10:1430. doi:10.3389/ fonc. 2020.01430

29. Zhu T, Ma Z, Wang H, et al. Immune-related long non-coding RNA signature and clinical nomogram to evaluate survival of patients suffering esophageal squamous cell carcinoma. Front Cell Dev Biol. 2021;9:641960. doi:10.3389/fcell.2021.641960

30. Ritchie ME, Phipson B, Wu D, et al. Limma powers differential expression analyses for RNA-sequencing and microarray studies. Nucleic Acids Res. 2015;43(7):e47. doi:10.1093/nar/gkv007
31. Kassambara A, Kosinski M, Biecek P, Fabian S. Package 'survminer'. In: Drawing Survival Curves Using 'Ggplot2'(R Package Version 03 1); 2017.

32. Mayakonda A, Lin D, Assenov Y, Plass C, Koeffler HP. Maftools: efficient and comprehensive analysis of somatic variants in cancer. Genome Res. 2018;28(11):1747-1756. doi:10.1101/gr.239244.118

33. Yi M, Nissley DV, Mccormick F, Stephens RM. SsGSEA score-based ras dependency indexes derived from gene expression data reveal potential ras addiction mechanisms with possible clinical implications. Sci Rep. 2020;10(1):1-16.

34. Hanzelmann S, Castelo R, Guinney J. GSVA: gene set variation analysis for microarray and RNA-seq data. BMC Bioinform. 2013;14(1):7. doi:10.1186/1471-2105-14-7

35. Wickham H. Ggplot2. Wiley Interdiscip Rev Comput Stat. 2011;3 (2):180-185. doi:10.1002/wics. 147

36. Team RC. R: a language and environment for statistical computing; 2013.

37. Sartor O, de Bono JS, Longo DL. Metastatic prostate cancer. New Engl J Med. 2018;378(7):645-657. doi:10.1056/ NEJMra1701695

38. Froehner M, Koch R, Graefen M, et al. Re: Nicolas Mottet, Roderick C.N. van den Bergh, Erik Briers, et al. EAU-EANM-ESTRO-ESURSIOG guidelines on prostate cancer-2020 update. Part 1: screening, diagnosis, and local treatment with curative intent. Eur Urol 2021;79:243-62. Eur Urol. 2021;79(5):e138. doi:10.1016/j. eururo.2021.02.005

39. Helleday T. PARP inhibitor receives FDA breakthrough therapy designation in castration resistant prostate cancer: beyond germline BRCA mutations. Ann Oncol. 2016;27(5):755-757. doi:10.1093/ annonc/mdw048

40. Shoag JE, Nyame YA, Gulati R, Etzioni R, Hu JC. Reconsidering the trade-offs of prostate cancer screening. New Engl J Med. 2020;382 (25):2465-2468. doi:10.1056/NEJMsb2000250

41. Rebello RJ, Oing C, Knudsen KE, et al. Prostate cancer. Nat Rev Dis Primers. 2021;7(1):9. doi:10.1038/s41572-020-00243-0

42. Wang Z, Leushkin E, Liechti A, et al. Transcriptome and translatome co-evolution in mammals. Nature. 2020;588(7839):642-647. doi:10.1038/s41586-020-2899-z

43. Cheng T, Huang S. Roles of non-coding RNAs in cervical cancer metastasis. Front Oncol. 2021;11:646192. doi:10.3389/ fonc.2021.646192

44. Parker KA, Robinson NJ, Schiemann WP. The role of RNA processing and regulation in metastatic dormancy. Semin Cancer Biol. 2021. doi:10.1016/j.semcancer.2021.03.020

45. Zhang C, Liang Y, Zhang C, et al. The novel role and function of LINC01235 in metastasis of gastric cancer cells by inducing epithelial-mesenchymal transition. Genomics. 2021;113 (3):1504-1513. doi:10.1016/j.ygeno.2021.03.027

46. Miao C, Bai L, Yang Y, Huang J. Dysregulation of lncRNAs in rheumatoid arthritis: biomarkers, pathogenesis and potential therapeutic targets. Front Pharmacol. 2021;12:652751. doi:10.3389/ fphar.2021.652751

47. Mu L, Ding K, Tu R, Yang W. Identification of 4 immune cells and a 5-lncRNA risk signature with prognosis for early-stage lung adenocarcinoma. J Transl Med. 2021;19(1):127. doi:10.1186/ s12967-021-02800-x

48. Yang L, Li A, Liu F, et al. Immune profiling reveals molecular classification and characteristic in urothelial bladder cancer. Front Cell Dev Biol. 2021;9:596484. doi:10.3389/fcell.2021.596484

49. Yan Y, Mao X, Zhang Q, et al. Molecular mechanisms, immune cell infiltration, and potential drugs for prostate cancer. Cancer Biomark. 2021:1-10. doi:10.3233/CBM-200939.

50. Shao N, Tang H, Mi Y, Zhu Y, Wan F, Ye D. A novel gene signature to predict immune infiltration and outcome in patients with prostate cancer. Oncoimmunology. 2020;9(1):1762473. doi:10.1080/ 2162402X.2020.1762473 
51. Wang Q, Ye Y, Yu H, et al. Immune checkpoint-related serum proteins and genetic variants predict outcomes of localized prostate cancer, a cohort study. Cancer Immunol Immunother. 2021;70 (3):701-712. doi:10.1007/s00262-020-02718-1

52. Qu L, Wang Z, Chen Q, et al. Prognostic value of a long non-coding RNA signature in localized clear cell renal cell carcinoma. Eur Urol. 2018;74(6):756-763. doi:10.1016/j.eururo.2018.07.032

53. Weiner AB, Vidotto T, Liu Y, et al. Plasma cells are enriched in localized prostate cancer in Black men and are associated with improved outcomes. Nat Commun. 2021;12(1):935. doi:10.1038/ s41467-021-21245-w

54. Subudhi SK, Vence L, Zhao H, et al. Neoantigen responses, immune correlates, and favorable outcomes after ipilimumab treatment of patients with prostate cancer. Sci Transl Med. 2020;12(537):z3577. doi:10.1126/scitranslmed.aaz3577

55. Galon J. Type, density, and location of immune cells within human colorectal tumors predict clinical outcome. Science. 2006;313 (5795):1960-1964. doi:10.1126/science.1129139

56. Nielsen JS, Sahota RA, Milne K, et al. CD20+ tumor-infiltrating lymphocytes have an atypical CD27-memory phenotype and together with $\mathrm{CD} 8+\mathrm{t}$ cells promote favorable prognosis in ovarian cancer. Clin Cancer Res. 2012;18(12):3281-3292. doi:10.1158/10780432.CCR-12-0234

57. Petitprez F, de Reyniès A, Keung EZ, et al. B cells are associated with survival and immunotherapy response in sarcoma. Nature. 2020;577(7791):556-560. doi:10.1038/s41586-019-1906-8

58. de Bono JS, Fleming MT, Wang JS, et al. Phase I trial of MEDI3726, a prostate-specific membrane antigen-targeted antibody-drug conjugate, in patients with mCRPC after failure of abiraterone or enzalutamide. Clin Cancer Res. 2021:2020-4528. doi:10.1158/ 1078-0432.CCR-20-4528.

59. Cha H, Lee JH, Ponnazhagan S. Revisiting immunotherapy: a focus on prostate cancer. Cancer Res. 2020;80(8):1615-1623. doi:10.1158/ 0008-5472.CAN-19-2948

60. Fay EK, Graff JN. Immunotherapy in prostate cancer. Cancers. 2020;12(7):1752. doi:10.3390/cancers 12071752

61. Hansen AR, Massard C, Ott PA, et al. Pembrolizumab for advanced prostate adenocarcinoma: findings of the KEYNOTE-028 study. Ann Oncol. 2018;29(8):1807-1813. doi:10.1093/annonc/mdy232

62. Passaro A, Stenzinger A, Peters S. Tumor mutational burden as a pan-cancer biomarker for immunotherapy: the limits and potential for convergence. Cancer Cell. 2020;38(5):624-625. doi:10.1016/j. ccell.2020.10.019
63. Sha D, Jin Z, Budczies J, Kluck K, Stenzinger A, Sinicrope FA. Tumor mutational burden as a predictive biomarker in solid tumors. Cancer Discov. 2020;10(12):1808-1825. doi:10.1158/2159-8290.CD20-0522

64. Hellmann MD, Callahan MK, Awad MM, et al. Tumor mutational burden and efficacy of nivolumab monotherapy and in combination with ipilimumab in small-cell lung cancer. Cancer Cell. 2018;33 (5):853-861. doi:10.1016/j.ccell.2018.04.001

65. Klein O, Kee D, Markman B, et al. Evaluation of TMB as a predictive biomarker in patients with solid cancers treated with anti-PD-1/CTLA-4 combination immunotherapy. Cancer Cell. 2021;39(5):592-593. doi:10.1016/j.ccell.2021.04.005

66. Rosenberg JE, Hoffman-Censits J, Powles T, et al. Atezolizumab in patients with locally advanced and metastatic urothelial carcinoma who have progressed following treatment with platinum-based chemotherapy: a single-arm, multicentre, Phase 2 trial. Lancet. 2016;387 (10031):1909-1920. doi:10.1016/S0140-6736(16)00561-4

67. Chan TA, Wolchok JD, Snyder A. Genetic basis for clinical response to CTLA-4 blockade in melanoma. $N$ Engl J Med. 2015;373 (20):1984. doi:10.1056/NEJMc1508163

68. de Bono J, Mateo J, Fizazi K, et al. Olaparib for metastatic castration-resistant prostate cancer. $N$ Engl J Med. 2020;382 (22):2091-2102. doi:10.1056/NEJMoa1911440

69. Claessens F, Visakorpi T. Endocrinology of prostate cancer. Mol Cell Endocrinol. 2018;462(Pt A):1-2. doi:10.1016/j.mce.2018.01.005

70. Shalapour S, Font-Burgada J, Di Caro G, et al. Immunosuppressive plasma cells impede T-cell-dependent immunogenic chemotherapy. Nature. 2015;521(7550):94-98. doi:10.1038/nature14395

71. Gebremeskel S, Lobert L, Tanner K, et al. Natural killer t-cell immunotherapy in combination with chemotherapy-induced immunogenic cell death targets metastatic breast cancer. Cancer Immunol Res. 2017;5(12):1086-1097. doi:10.1158/2326-6066.CIR-17-0229

72. Emens LA, Middleton G. The interplay of immunotherapy and chemotherapy: harnessing potential synergies. Cancer Immunol Res. 2015;3(5):436-443. doi:10.1158/2326-6066.CIR-15-0064

73. Ramakrishnan R, Gabrilovich DI. Mechanism of synergistic effect of chemotherapy and immunotherapy of cancer. Cancer Immunol Immunother. 2011;60(3):419-423. doi:10.1007/s00262-010-0930-1

74. Hurwitz M. Chemotherapy in prostate cancer. Curr Oncol Rep. 2015;17(10):44. doi:10.1007/s11912-015-0468-7
International Journal of General Medicine

\section{Publish your work in this journal}

The International Journal of General Medicine is an international, peer-reviewed open-access journal that focuses on general and internal medicine, pathogenesis, epidemiology, diagnosis, monitoring and treatment protocols. The journal is characterized by the rapid reporting of reviews, original research and clinical studies across all disease areas. The manuscript management system is completely online and includes a very quick and fair peer-review system, which is all easy to use. Visit http://www.dovepress.com/ testimonials.php to read real quotes from published authors. 